\title{
High-Fat Diet-Induced Functional and Pathologic Changes in Lacrimal Gland
}

Xin He, ${ }^{* \dagger}$ Zhongyang Zhao, ${ }^{* \dagger \ddagger}$ Shaopan Wang, ${ }^{* \dagger \ddagger}$ Jie Kang, ${ }^{* \dagger \ddagger}$ Minjie Zhang, ${ }^{* \dagger \ddagger}$ Jinghua Bu, ${ }^{* \dagger \ddagger}$ Xiaoxin Cai, ${ }^{* \dagger \ddagger}$ Changkai Jia, ${ }^{* \dagger}$ Yixuan $\mathrm{Li}^{\dagger}{ }^{\dagger}$ Kechun Li, ${ }^{\top}$ Peter Sol Reinach, Andrew J. Quantock, ${ }^{* *}$

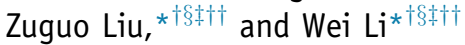

From the Eye Institute of Xiamen University, ${ }^{*}$ the School of Medicine, ${ }^{\dagger}$ the Xiang'an Hospital of Xiamen University, ${ }^{\S}$ and the Fujian Provincial Key Laboratory of Ophthalmology and Visual Science, ${ }^{\ddagger}$ Xiamen University, Xiamen, China; Datong Middle School, ${ }^{\uparrow}$ Xiamen, China; the Xiamen University Affiliated Xiamen Eye Center ${ }^{\dagger \dagger}$ Xiamen, China; Wenzhou Medical University," Wenzhou, China; and the School of Optometry and Vision Sciences, ${ }^{* *}$ Cardiff University, Cardiff, United Kingdom

Accepted for publication September 3, 2020.

Address correspondence to Zuguo Liu, M.D., Ph.D., or Wei Li, M.D., Eye Institute of Xiamen University, Chengyi Building, 4th Floor, 4221-122, South Xiang'an Rd, Xiamen, Fujian, 361102, China. E-mail: zuguoliu@xmu.edu.cn or wei1018@xmu.edu.cn.

\begin{abstract}
The lacrimal gland is critical for maintaining the homeostasis of the ocular surface microenvironment through secreting aqueous tears in mammals. Many systemic diseases such as Sjögren syndrome, rheumatoid arthritis, and diabetes can alter the lacrimal gland function, eventually resulting in aqueous tear-deficient dry eye. Here, a high-fat diet (HFD) experimental mouse model was used to clarify how hyperlipidemia affects lacrimal gland function. Aqueous tear secretion fell about $50 \%$ after 1 month on a HFD. Lipid droplets accumulated in the matrix and acinar cells of the lacrimal gland after this period, along with changes in the lipid metabolism, changes in gene expression levels, and disruption of fatty acid oxidative activity. Immune cell infiltration and rises in the gene expression levels of the inflammation-related cytokines Il1 $\beta, \operatorname{Tnf} \alpha$, Tsg6, Il10, Mmp2, and Mmp9 were found. HFD also induced mitochondrial hypermegasoma, increased apoptosis, and decreased lacrimal gland acinar cell proliferation. Replacement of the HFD with the standard diet partially reversed pathologic changes in the lacrimal gland. Similarly, supplementing the HFD with fenofibrate also partially reversed the inhibited tear secretion and reduced lipid accumulation, inflammation, and oxidative stress levels. The authors conclude that a HFD induces pathophysiological changes and functional decompensation of the lacrimal gland. Therefore, ingestion of a HFD may be a causative factor of dry eye disease. (Am J Pathol 2020, 190: 2387-2402; https://doi.org/10.1016/j.ajpath.2020.09.002)
\end{abstract}

The lacrimal gland secretes aqueous tears onto the anterior ocular surface that are critical for the maintenance of ocular surface homeostasis and health. ${ }^{1-3}$ Declines in its function can lead to aqueous tear-deficient dry eye. ${ }^{4}$ A variety of causes, including autoimmune diseases such as Sjögren syndrome, 5,6 allogeneic hematopoietic cell transplantation, ${ }^{7}$ ligation of the lacrimal gland duct, ${ }^{8}$ and aging ${ }^{9}$ could induce lacrimal gland damage. These injuries could result in lymphocytic infiltration, fibrosis, and acinar cell atrophy of the lacrimal gland. Any of these changes may induce sufficient lacrimal gland malfunction to cause dry eye disease. ${ }^{10}$

In addition to the aforementioned pathophysiological conditions that may alter lacrimal gland structure and function, the possible impact of other systemic abnormalities is progressively attracting more attention. For example, it was shown that lipid droplet accumulation in the lacrimal gland acinar increases with age in a non-obese diabetic (NOD) mouse model. This change is accompanied by lymphocytic infiltration and destruction of the acini. Furthermore, lacrimal gland cholesteryl esters significantly increased in these mice. ${ }^{11}$ In the authors' sleep deprivation-induced mouse dry eye model, abnormal lipid metabolism and lipid accumulation were also found in the lacrimal gland. ${ }^{12}$ In these different animal models, the authors became aware of a certain association between

Supported in part by the National Key R\&D Program of China grants 2018YFA0107301 (W.L.) and 2018YFA0107304 (Z.L.); and the National Natural Science Foundation of China (NSFC) grants 81970773, 81770894 (W.L.), and 81870627 (Z.L.).

Disclosures: None declared. 
aberrant lipid metabolism and dry eye disease. Populationbased clinical studies are supportive of this notion because dry eye patients were more likely to have the comorbidity of hyperlipidemia. ${ }^{13-16}$ Recently, the authors found that hyperlipidemia in apolipoprotein E knockout $\left(\mathrm{ApoE}^{-/-}\right)$mice induced exaggerated lipid accumulation, abnormal keratinization, and inflammatory cell infiltration in the meibomian gland and surrounding microenvironment, resulting in meibomian gland dysfunction, a contributing pathophysiological condition in evaporative dry eye disease. ${ }^{17}$

Although there is an indication that lipid metabolism in the lacrimal gland is related to its functional status, there are no studies available describing the direct effect of hyperlipidemia on this question. Specifically, it is unknown whether systemic abnormal lipid metabolism disrupts lacrimal gland function. In the current study, the authors dealt with this question by characterizing the effects of hyperlipidemia on lacrimal gland morphology and function in a high-fat diet (HFD) experimental mouse model. The results show that the HFD induced abnormal lipid accumulation, inflammation, lipid peroxidation, and apoptosis in the lacrimal gland, which led to declines in aqueous tear secretion.

\section{Material and Methods}

\section{HFD Mouse Model and Treatments}

Male C57BL/6 mice were obtained from Shanghai SLAC laboratory animal center (Shanghai, China). All animals were kept in ventilated cages in an animal care facility at $25^{\circ} \mathrm{C} \pm 1{ }^{\circ} \mathrm{C}$, relative humidity $60 \% \pm 10 \%$, and on alternating 12-hour light-dark cycles (8 AM to $8 \mathrm{PM}$ ). All experimental procedures were performed in accordance with the Association for Research in Vision and Ophthalmology Statement for the Use of Animals in Ophthalmic and Vision Research. The experimental animal ethics committee of Xiamen University approved the experimental protocol. The mice were fed a standard diet (StD; $10 \mathrm{kcal} \%$ fat) or a HFD (60 kcal\% fat) beginning at the age of 4 weeks and extending from 1 to 4 months. Another group was at first fed the HFD for 1 month and then switched to the StD for another 1 month. A third group was at first fed the HFD for 4 months, which was then supplemented with $100 \mathrm{mg} / \mathrm{kg}$ per day of fenofibrate for another month. Following sacrifice, their exorbital lacrimal glands were harvested for different experimental studies.

\section{Measurement of Aqueous Tear Secretion}

Tear production was measured daily at $3 \mathrm{PM}$ with phenol red cotton threads (Zone-Quick; Yokota, Tokyo, Japan). Before each measurement, absorbent paper was used to remove the tear fluid inside the conjunctival sac to avoid any contribution from the lacrimal lake. a thread was then placed in the lower conjunctival fornix for 15 seconds at a position that was onethird of the distance between the lower eyelid and the lateral canthus. The distance that the red color dye front migrated along the thread length was recorded in millimeters.

\section{Transmission Electron Microscopy}

Mice were sacrificed and their lacrimal glands were removed immediately and then briefly washed with phosphate-buffered saline (PBS). The tissues were cut into $1-\mathrm{mm}^{3}$ blocks and first fixed in pre-chilled $2.5 \%$ glutaraldehyde. They were then stored overnight at $4^{\circ} \mathrm{C}$, washed three times with sodium cacodylate buffer, and post-fixed in osmium tetroxide for 1.5 hours at $4^{\circ} \mathrm{C}$. The tissue was then serially dehydrated in $30 \%$ and $50 \%$ ethanol, and stained in $70 \%$ uranyl acetate for 4 hours, followed by serial dehydration in $70 \%, 90 \%$, and $100 \%$ ethanol. The tissue was then immersed in Spurr resin and sectioned into $75-\mathrm{nm}$ slices and stained with lead citrate for 10 minutes. Images were captured with a Tecnai G2 Spirit Bio Twin electron microscope (FEI Company, Hillsboro, OR).

\section{Oil Red 0 Staining}

All cryosections (6- $\mu$ m thickness) were covered with $4 \%$ paraformaldehyde for 5 minutes and subsequently rinsed with PBS. The sections were stained with filterable Oil Red $\mathrm{O}$ solution $(0.5 \%$ Oil Red O in isopropanol) and co-dyed with hematoxylin. After rinsing in PBS, the samples were mounted in glycerin. The lipid droplets were observed under a microscope.

\section{Cholesterol Assay}

The cholesterol assay kit (ab65390; Abcam, Cambridge, MA) was used to determine lacrimal gland cholesterol content. Lacrimal gland tissues were briefly washed with cold PBS and weighed. One hundred microliters of the cholesterol assay buffer were added to each sample that had been cut into small pieces. They were centrifuged at $17,900 \times g$ for 10 minutes, and the supernatant was recovered. It was aliquoted into the cholesterol reaction mix and incubated at $37^{\circ} \mathrm{C}$ for 1 hour in subdued illumination. The results were measured on a microplate reader at OD $570 \mathrm{~nm}$.

\section{Real-Time PCR}

Total RNA of the lacrimal gland was extracted in TRIzol (Invitrogen, Carlsbad, CA), and the ExScript RT Reagent kit (Takara Bio, Otsu, Shiga, Japan) was used to reverse transcribe it into cDNA. Real-time PCR was performed with a Roche Real-Time PCR detection system according to the manufacturer's instructions (Roche, Basel, Switzerland) following addition of the Hieff qPCR SYBR Green Master Mix (Yeasen Bio, Shanghai, China). The amplification program included a preincubation step at $95^{\circ} \mathrm{C}$ for 600 seconds, followed by denaturation at $95^{\circ} \mathrm{C}$ for 10 seconds, annealing at $60^{\circ} \mathrm{C}$ for 10 seconds, and extension at $72^{\circ} \mathrm{C}$ for 
20 seconds, for 45 cycles. The primers used to amplify specific gene products are shown in Table 1. Results were calculated according to the $2^{-\Delta \Delta C T}$ method $^{18}$ to evaluate relative gene expression levels.

\section{Immunofluorescence Staining}

After isolating the lacrimal gland tissue, pre-cooled PBS was used to wash adherent blood. Filter paper was then used to wipe off any remaining liquid from the tissue. The tissue was placed into an embedding box to which was added OCT compound to immerse all of the tissue. The box was then placed into liquid nitrogen for 1 minute and stored at $-80^{\circ} \mathrm{C}$ to obtain frozen sections. Lacrimal gland cryosections (6- $\mu \mathrm{m}$ thick) were fixed in $4 \%$ paraformaldehyde for 20 minutes at room temperature. After washing in PBS, the sections were incubated in $0.2 \%$ Triton X-100 for $20 \mathrm{mi}-$ nutes. Subsequently, they were first incubated with $2 \%$ bovine serum albumin for 60 minutes and then covered with anti-F4/80 (1:100, ab6640; Abcam) or anti-alpha smooth muscle actin ( $\alpha$-SMA, 1:200, ab32575; Abcam), anti-Ki67 (1:200, ab16667; Abcam), anti-K19 (1:200, ab52625; Abcam) overnight at $4{ }^{\circ} \mathrm{C}$. After washing three times in PBS for 10 minutes each, the sections were covered with Alexa Fluor 594-conjugated IgG (1:300, R37119; Life Technologies, Carlsbad, CA) or Alexa Fluor 488-conjugated IgG (1:300, A-21208, A21206; Life Technologies) for $60 \mathrm{mi}-$ nutes at room temperature, followed by three sequential washes in PBS for 10 minutes each. DAPI was used to stain the sections (Vector Laboratories, Burlingame, CA), and then a laser confocal microscope (Olympus FluoView 1000; Olympus, Tokyo, Japan) was used to view them.

\section{Immunohistochemical Staining}

Paraffin sections (4- $\mu \mathrm{m}$ thick) were immersed in xylene for 20 minutes followed by serial dewaxing in $100 \%$, 95\%, $90 \%$, and $80 \%$ ethanol. After dewaxing, the sections were washed with PBS for 5 minutes and then put into the antigen retrieval solution $(10 \mathrm{mmol} / \mathrm{L}$ sodium citrate, $0.05 \%$ Tween20, pH 6.0, or Tris-EDTA buffer, pH 9.0) and boiled for 20 minutes in a microwave. Following cooling, the sections were rinsed in running water for 20 minutes. They were then washed with PBS after being incubated in $0.6 \%$ $\mathrm{H}_{2} \mathrm{O}_{2}$ for 30 minutes. Afterward, they were incubated in $0.2 \%$ Triton X-100 for 20 minutes. After washing three more times with PBS and incubating with $2 \%$ bovine serum albumin for 60 minutes, they were then covered with anti-4-hydroxyalkenal (4HNE; 1:200, ab46545; Abcam), anti-CD4 (1:1000, ab183685; Abcam), or anti-Ly6g (1:2000, ab238132; Abcam) overnight at $4^{\circ} \mathrm{C}$. After three more washings for 10 minutes each with PBS, the sections were covered with anti-rabbit secondary antibody (PV6001; ZSGB-BIO, Beijing, China) for 60 minutes at room temperature, followed by another three PBS washes for 10 minutes each. A DAB Kit (ZLI9019; ZSGB-BIO) was used for visualization and costaining with hematoxylin, followed by serial dehydration in $70 \%, 80 \%, 95 \%$, and $100 \%$ ethanol.

\section{Malondialdehyde Detection Assay}

The MDA kit (A003-1; Nanjing Jiancheng Bioengineering Institute, Nanjing, China) was applied to measure malondialdehyde (MDA) content in the lacrimal gland. Tissue isolates were briefly washed with cold PBS and placed into chilled $0.9 \%$ saline. Tissue homogenate of small pieces was mixed with the kit contents according to the manufacturer's instructions. After incubating at $95^{\circ} \mathrm{C}$ for 40 minutes, the results were measured on a microplate reader at OD $532 \mathrm{~nm}$.

\section{In Situ Cell Apoptosis Detection Assay}

To measure the end-stage apoptosis, terminal deoxynucleotidyl transferase-mediated dUTP nick-end labeling

Table 1 Mouse Sequences Used for Quantitative Real-Time PCR

\begin{tabular}{|c|c|c|}
\hline Gene & Forward primer & Reverse primer \\
\hline Actb & $5^{\prime}$-CACCCGCGAGTACAACCTTC-3' & $5^{\prime}-\mathrm{CCCATACCCACCATCACACC-3^{ \prime }}$ \\
\hline Cpt1a & $5^{\prime}$-CTTCCAACGCATGACAGCAC- $3^{\prime}$ & $5^{\prime}-$ TTAACCATGATCGGCCCTCG-3' \\
\hline Ppar $\alpha$ & $5^{\prime}$-GAACCGGAACAAATGCCAGT-3' & 5'-CTTCAGGTAGGCTTCGTGGA-3' \\
\hline$N f e 2 l 2$ & $5^{\prime}-\mathrm{ATCCAGACAGACACCAGTGGA}-3^{\prime}$ & $5^{\prime}-$ CTGATGAGGGGCAGTGAAGAC- $3^{\prime}$ \\
\hline Cat & 5'-GCGGATTCCTGAGAGAGTGG-3' & 5'-АTTTCACTGCAAACCCCCGA-3' \\
\hline $\operatorname{Tnf} \alpha$ & $5^{\prime}-\mathrm{AGCCCACGTAGCAAACCACCAA-3^{ \prime }}$ & $5^{\prime}$-АСАСССАTTCССТTCACAGAGCAAT-3' \\
\hline Il1 $\beta$ & 5'-ATGCCACCTTTTGACAGTGATG-3' & $5^{\prime}-\mathrm{AGCTTCTCCACAGCCACAAT-3^{ \prime }}$ \\
\hline Tsg6 & 5'-AGCAGACCTGGTTGTCATCG-3' & $5^{\prime}$-AATCCGGCTCAACAGGAGTG-3' \\
\hline Il10 & $5^{\prime}$-ACAGCCGGGAAGACAATAACT-3' & $5^{\prime}$-TGATTTCTGGGCCATGCTTC-3' \\
\hline Mmp2 & 5'-GTGTAGATCGGGGCCATCAG-3' & 5'-CCCCATGTGTCTTCCCCTTC-3' \\
\hline
\end{tabular}


A

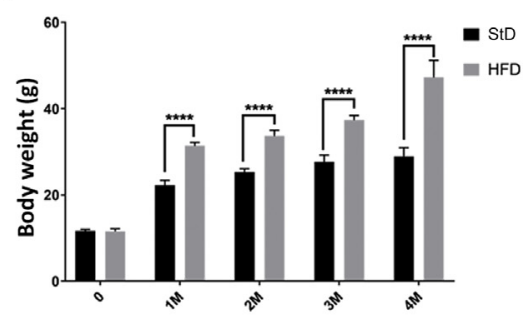

B

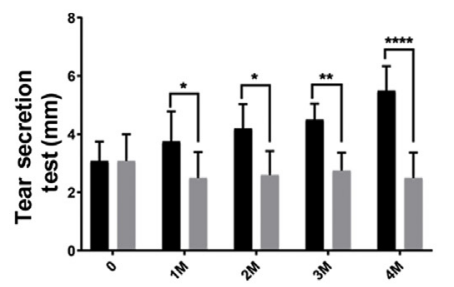

C

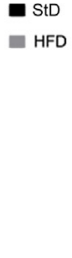

D

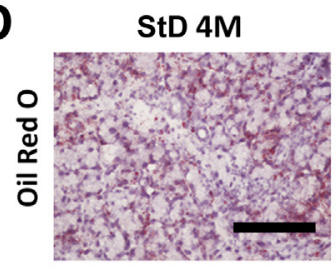

E

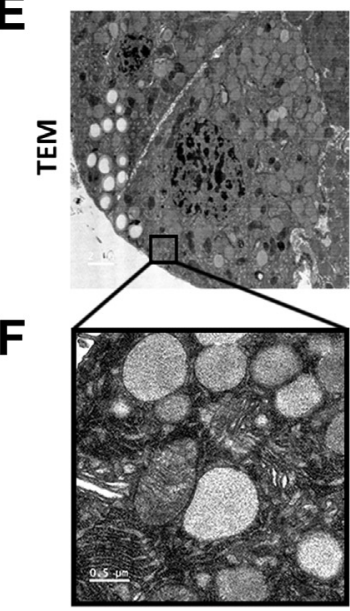

G

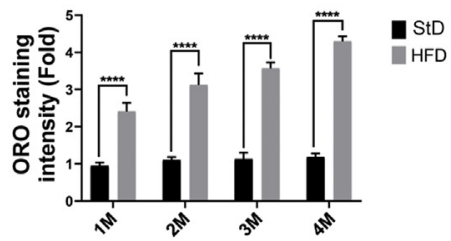

J

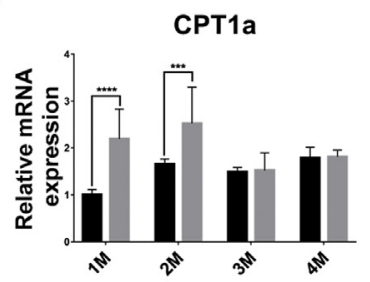

HFD 1M

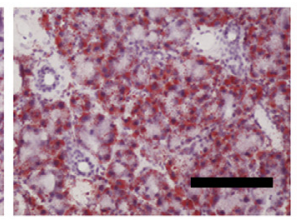

HFD 2M

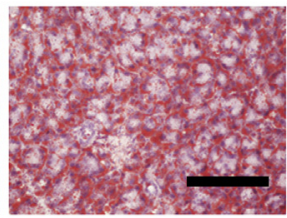

HFD 3M
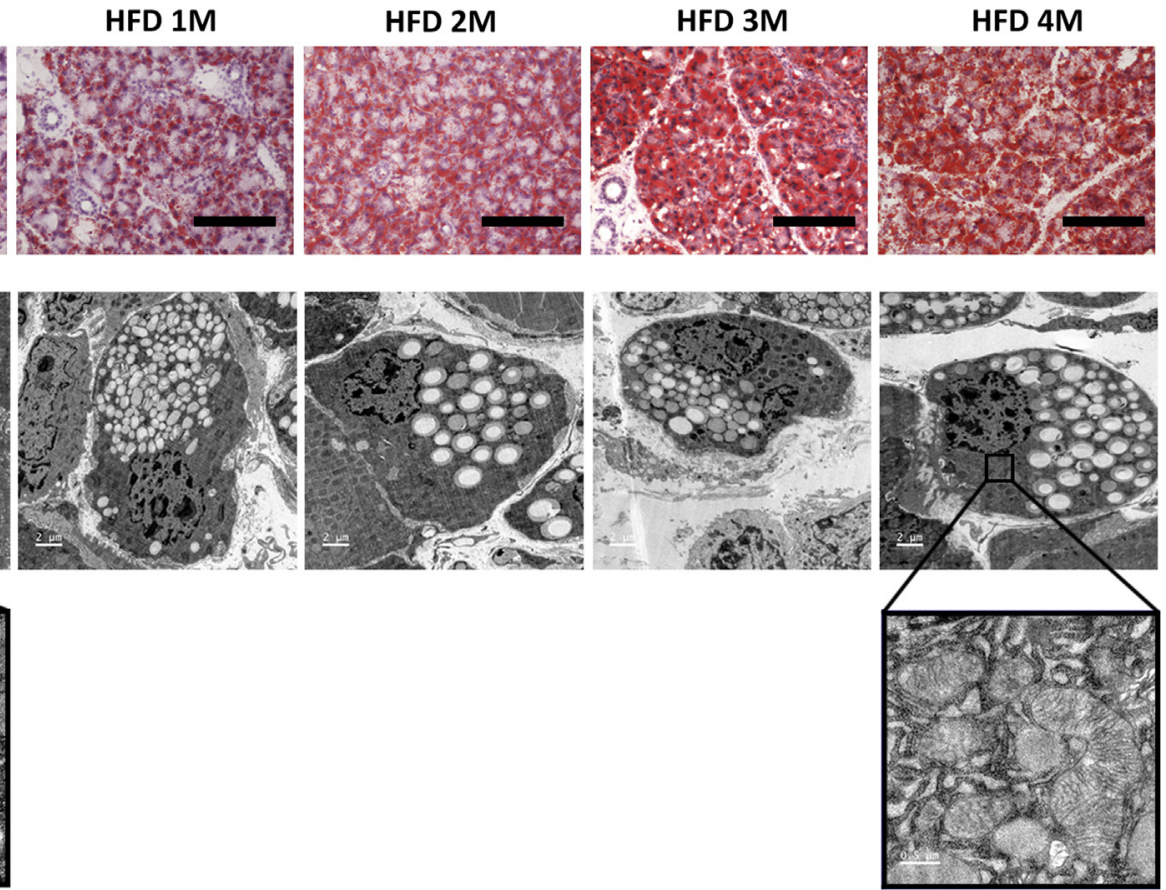

H

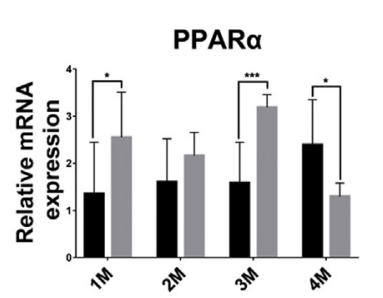

\section{I}

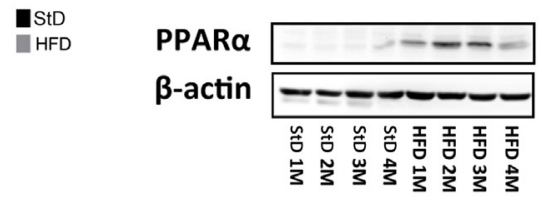

K

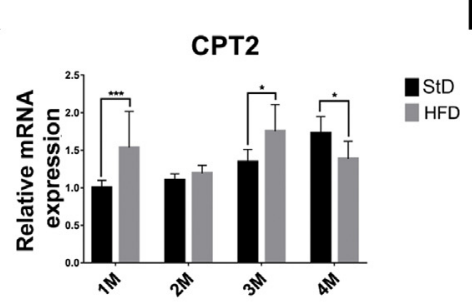

Cholesterol

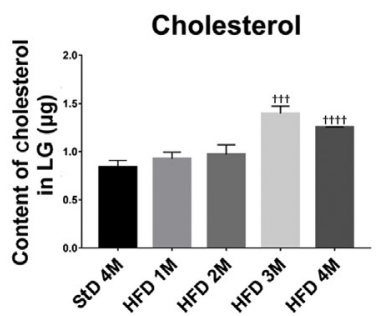

Figure 1 Tear secretion decrease and abnormal lipid metabolism of the lacrimal gland in high-fat diet (HFD) mice. A: Body weight was examined throughout the experiment. B: Tear secretion was measured by phenol red thread test. C: Lacrimal gland weight was examined throughout the experiment. D: 0il Red 0 staining shows lipid accumulation in the lacrimal gland. E: Transmission electron microscopy (TEM) shows the ultrastructure of the acinar cells of the lacrimal gland. Boxed areas are shown at higher magnification in F. F: TEM shows the ultrastructure of mitochondria in acinus after 4 months (4M) of HFD. G: 0il Red 0 (OR0) staining intensity was analyzed by ImageJ software version 1.53a. $\mathbf{H}$ and I: Real-time PCR analysis and Western blot analysis show Ppar $\alpha$ expression. J and K: Real-time PCR analyzed gene expression of Cpt1a, Cpt2. L: Cholesterol assay kit shows the content of total cholesterol in the lacrimal gland (LG). $n=5$ (A-C and $\mathbf{G}-\mathbf{L}) ; n=3(\mathbf{D}-\mathbf{F})$. Data are expressed as means \pm SD. ${ }^{*} P<0.05,{ }^{* *} P<0.01,{ }^{* *} P<0.001$, and ${ }^{* * * *} P<0.0001$ (one-way analysis of variance); ${ }^{\dagger \dagger \dagger} P<0.001,{ }^{\dagger \dagger \dagger} P<0.0001$ versus StD 4M (independent sample $t$-test). Scale bars: $100 \mu \mathrm{m}$ (D); $2 \mu \mathrm{m}(\mathbf{E}) ; 0.5 \mu \mathrm{m}$ (F). StD, standard diet. 
A

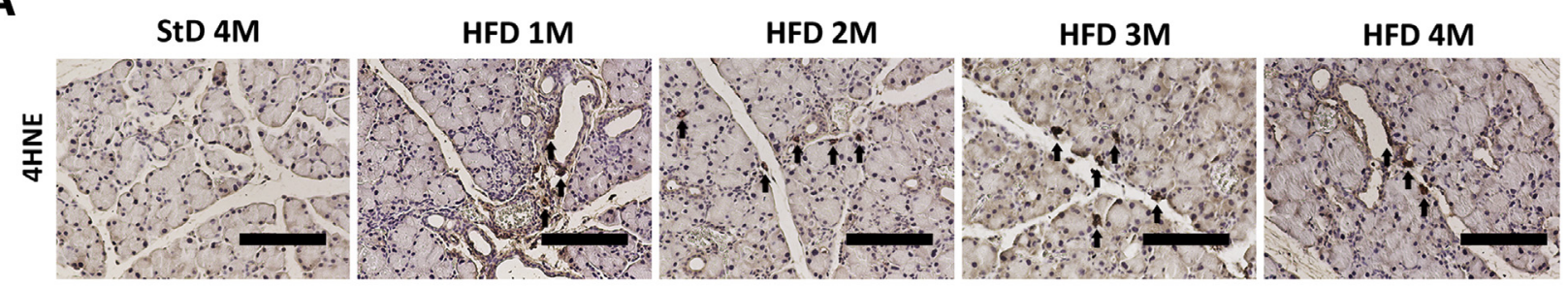

B

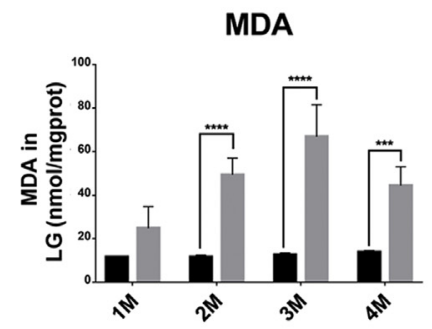

D

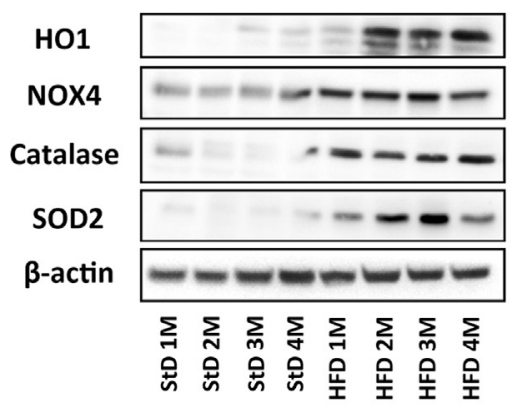

C
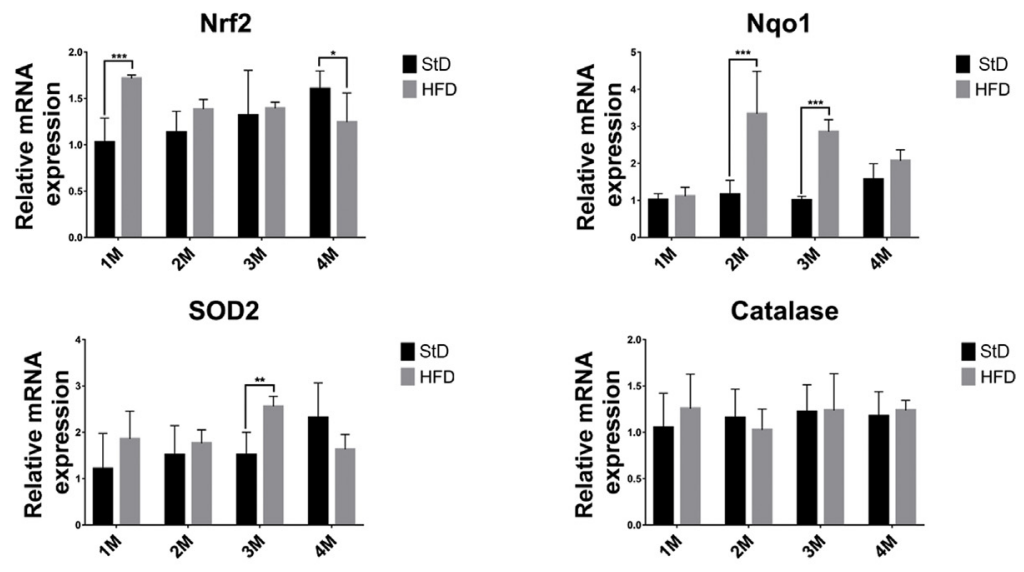

$\mathbf{E}$
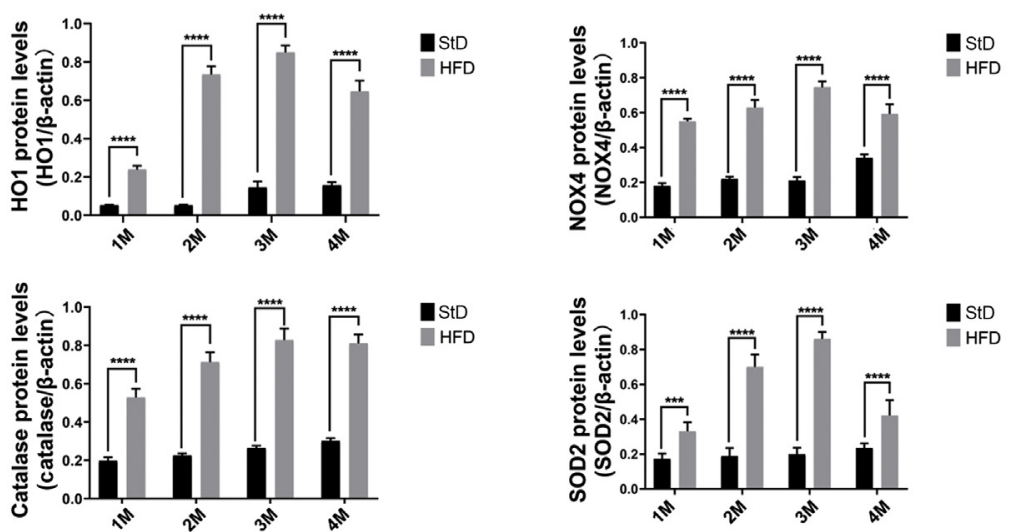

Figure 2 Lipid peroxidation of the lacrimal gland in high-fat diet (HFD) mice. A: Immunohistochemical staining of 4HNE shows the level of lipid peroxidation in the lacrimal gland after HFD. The arrows indicate positive staining. B: MDA kit detected the degree of malondialdehyde in the lacrimal gland (LG). C: Real-time PCR analyzed the gene expression of Nrf2, Nqo1, catalase, and Sod2. D and E: Expression of Ho1, Nox4, catalase, Sod2, and $\beta$-actin were examined by Western blot analysis. $n=3(\mathbf{A}) ; n=5$ (B-E). ${ }^{*} P<0.05,{ }^{* *} P<0.01,{ }^{* *} P<0.001$, and ${ }^{* * * *} P<0.0001$ (one-way analysis of variance). Scale bars $=100 \mu \mathrm{m}$. M, month; StD, standard diet.

(TUNEL) staining was performed to evaluate time-dependent changes in the lacrimal gland nuclear configuration. The frozen sections were incubated with $4 \%$ paraformaldehyde for 10 minutes followed by three PBS washes for 5 minutes each. All sections were then covered with $20 \mu \mathrm{g} / \mathrm{mL}$ proteinase-K for 15 minutes at room temperature. After three washings with PBS for 5 minutes each, the sections were incubated with assay (DeadEnd Fluorometric TUNEL System G3250; Promega, Madison, WI) according to the manufacturer's instructions for 1 hour in $37^{\circ} \mathrm{C}$. Then $2 \times$ saline sodium citrate was used to terminate the reaction. Tissues were then rinsed three times in
PBS (5 minutes per rinse) and were counterstained with DAPI, mounted, and then photographed with the use of a confocal laser scanning microscope (FluoView 1000; Olympus).

\section{Western Blot Analysis}

Lacrimal gland tissue was extracted with RIPA Lysis Buffer (Sigma-Aldrich, St. Louis, MO) containing protease inhibitor cocktails (Thermo Fisher Scientific, Waltham, MA) on ice for 30 minutes. Lysates were centrifuged, and the protein concentration of the supernatant was determined using a BCA 
A
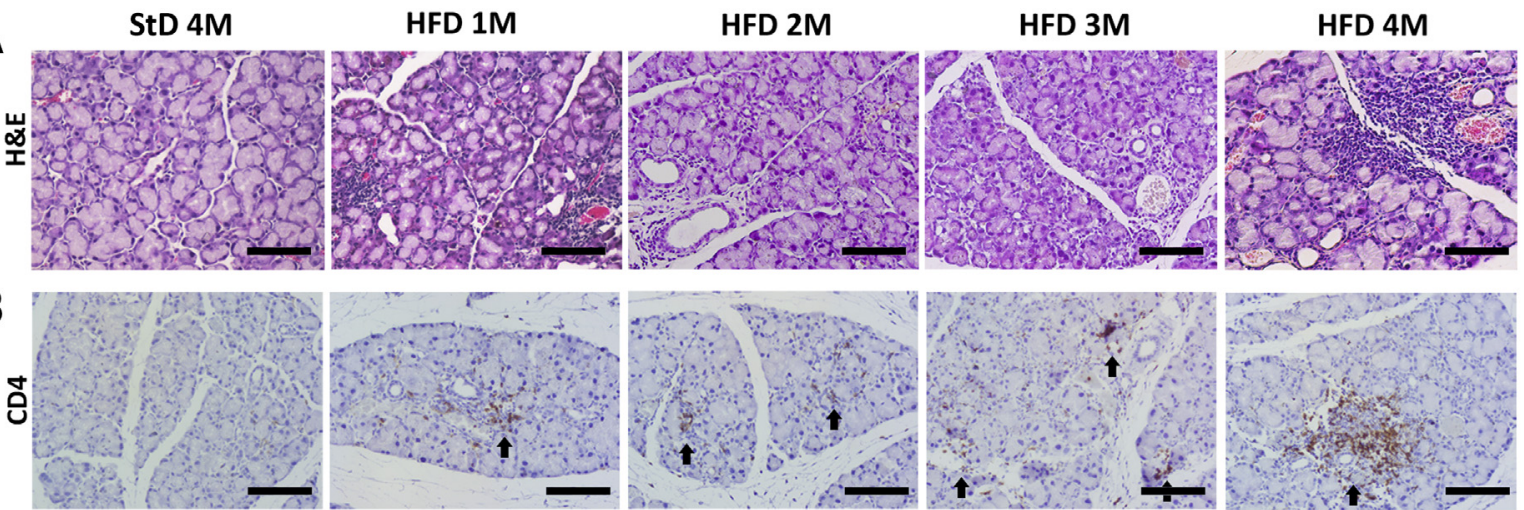

C
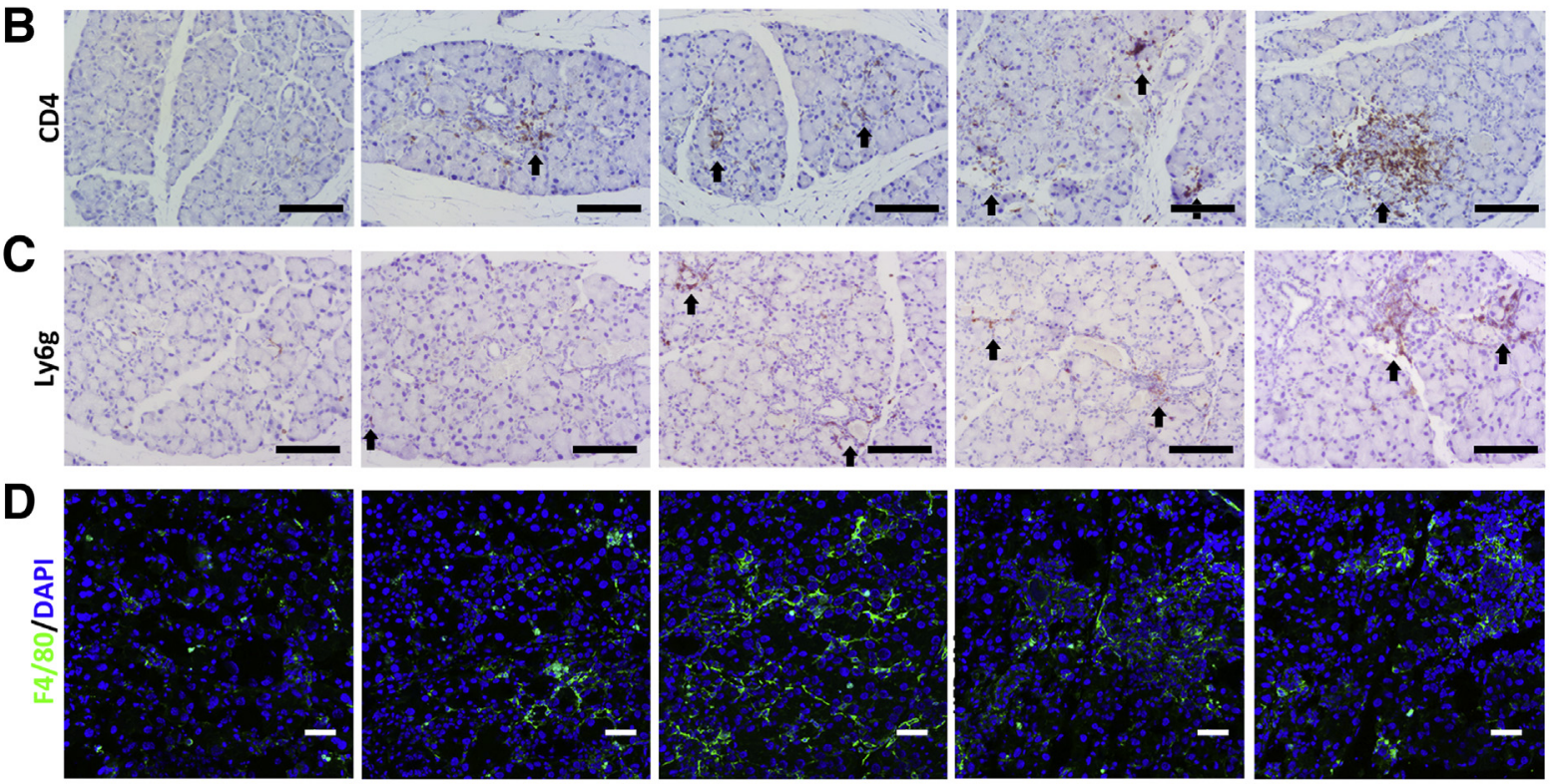

E

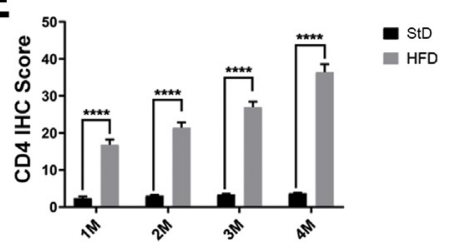

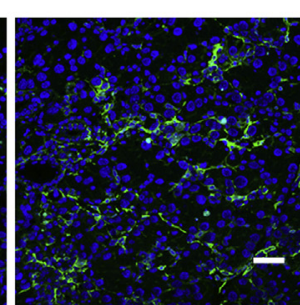

F
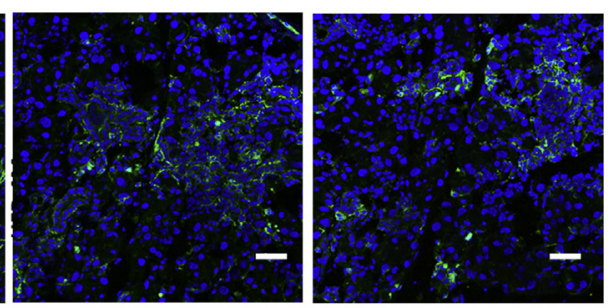

G

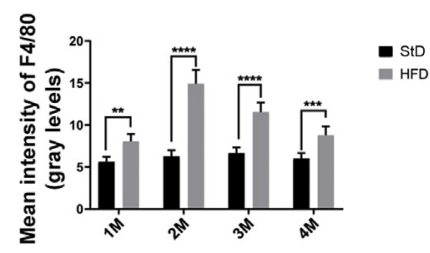

H

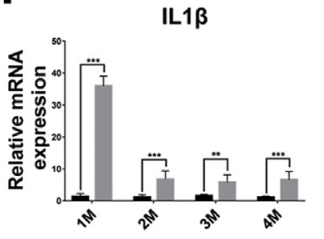

J

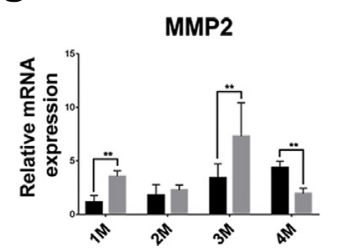

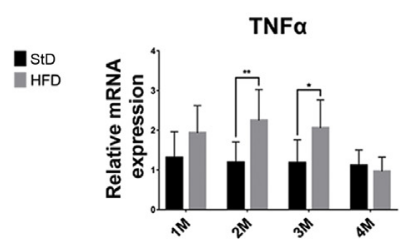

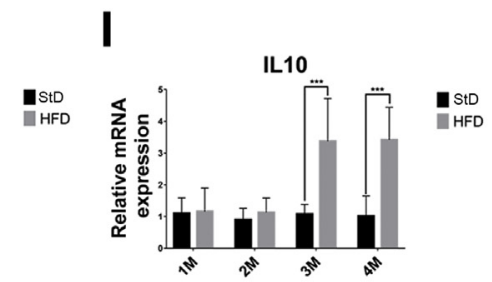

K

Figure 3 High-fat diet (HFD) induced inflammation in the lacrimal gland. A: Hematoxylin and eosin (H\&E) staining shows morphology changes of the lacrimal gland after HFD at different time points. B: Immunohistochemical staining of CD4 reveals CD4 ${ }^{+} \mathrm{T}$ cells in the lacrimal gland after HFD. Arrows indicate positive staining. C: Immunohistochemical staining of Ly6g shows neutrophils in the lacrimal gland. Arrows indicate positive staining. D: Immunofluorescence staining of F4/80 shows macrophages in the lacrimal gland (green: F4/80, blue: DAPI). E: ImageJ IHC Profile (Molecular Functional Imaging Laboratory, Navi Mumbai, India) analysis shows the CD4-positive score. F: ImageJ IHC Profile analysis shows the Ly6g-positive score. G: Mean fluorescence intensity of F4/80 was analyzed. H: Real-time PCR results exhibit proinflammatory factors Il11 $\beta$, Tnf $\alpha$. I: Real-time PCR results show antiinflammatory factors Il10, Tsg6. J: Real-time PCR analyzed gene expression of Mmp2 and Mmp9 in the lacrimal gland. K: Western blot results show the degree of Mmp9 expression in the lacrimal gland. $n=3(\mathbf{A}-\mathbf{D}) ; n=5(\mathbf{E}-\mathbf{K}) .{ }^{*} P<0.05,{ }^{* *} P<0.01,{ }^{* *} P<0.001$, and ${ }^{* * * *} P<0.0001$ (one-way analysis of variance). Scale bars: $100 \mu \mathrm{m}(\mathbf{A}-\mathbf{C}) ; 40 \mu \mathrm{m}$ (D). M, month; StD, standard diet. 
A
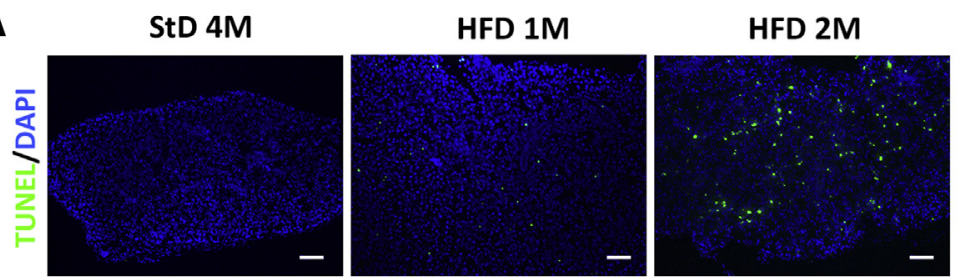

HFD 3M

HFD 4M

B
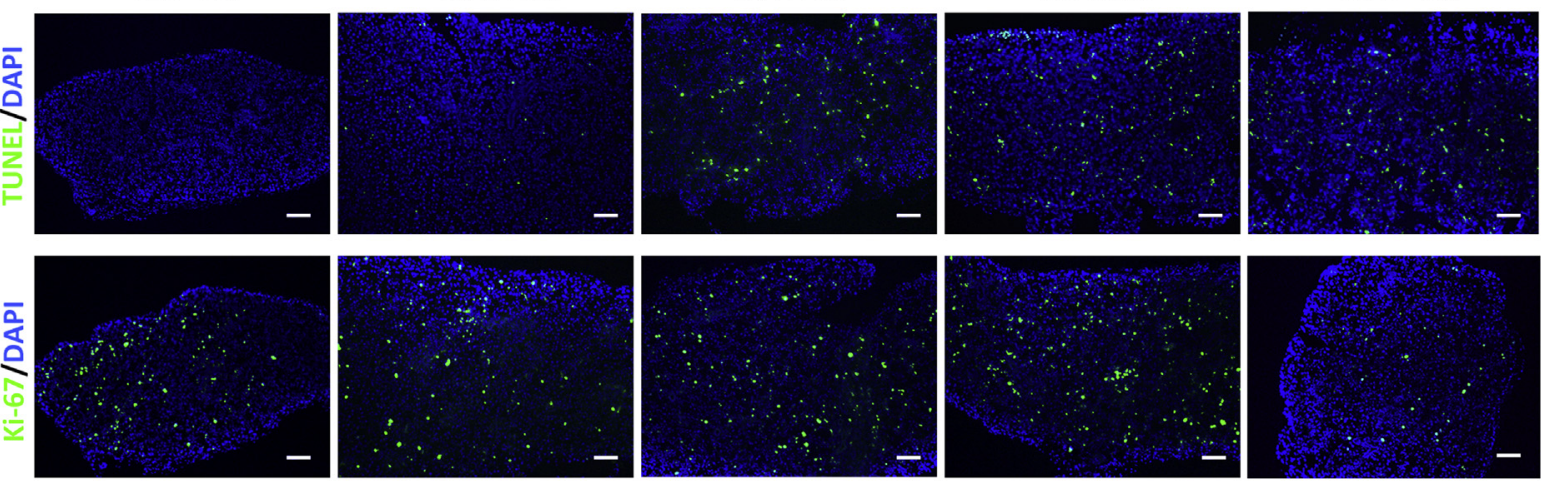

C
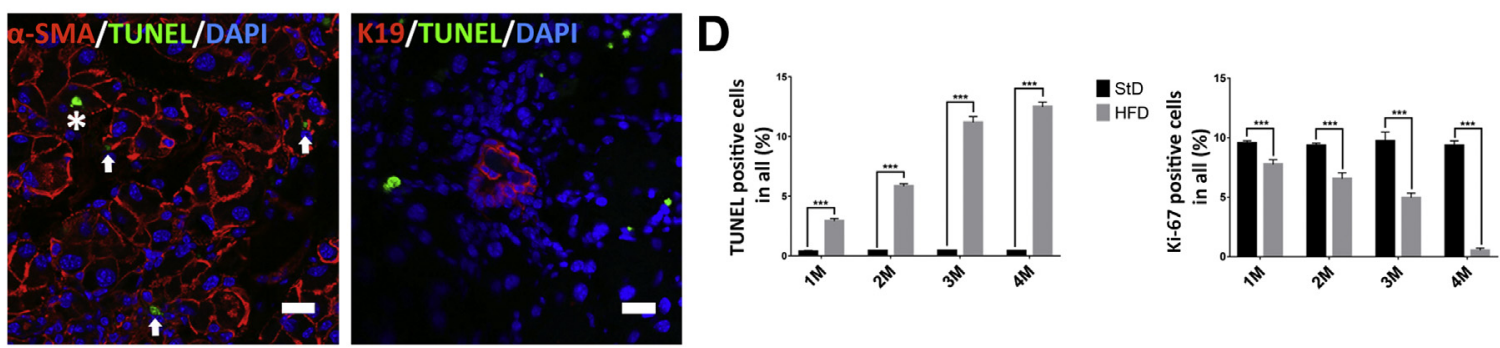

- StD

Figure 4 Apoptosis and proliferation changes of the lacrimal gland in high-fat diet (HFD) mice. A: TUNEL staining shows apoptotic cells in the lacrimal gland (green: TUNEL, blue: DAPI). B: Immunofluorescent staining of Ki-67 shows proliferative cells in the lacrimal gland (green: Ki-67, blue: DAPI). C: Immunofluorescence staining of $\alpha$-SMA reveals myoepithelial cells (red: $\alpha$-SMA, green: TUNEL, blue: DAPI, arrows indicate myoepithelial cells, asterisk indicates acinar cell). Immunofluorescence staining of K19 shows ducts of the lacrimal gland (red: K19, green: TUNEL, blue: DAPI). D: Graphs show the specific counts of positive cells by TUNEL staining and Ki-67-positive staining cells. $n=5$ (A-D). ${ }^{* *} P<0.001$ (one-way analysis of variance). Scale bar: $200 \mu$ m (A and B); $20 \mu \mathrm{m}$ (C). M, month; StD, standard diet; TUNEL, terminal deoxynucleotidyl transferase-mediated dUTP nick-end labeling.

Protein Assay kit (Thermo Fisher Scientific). Twenty micrograms of protein extracts were resolved by electrophoresis on $10 \%$ SDS-PAGE and then electrotransferred to a polyvinylidene difluoride membrane. After blocking in $2 \%$ bovine serum albumin for 1 hour, the membranes were probed overnight at $4{ }^{\circ} \mathrm{C}$ with primary antibodies to PPAR $\alpha(1: 1000$, ab8934; Abcam), heme oxygenase 1 (HO1; 1:1000, ab13243; Abcam), NADPH oxidase 4 (1:1000, ab195301; Abcam), catalase (1:1000, ab16731; Abcam), SOD2 (1:1000, ab13533; Abcam), matrix metalloproteinase 9 (MMP9; 1:800, ab38898; Abcam), $\alpha$-SMA (1:1000, ab124964; Abcam), and $\beta$-actin (1:10,000, A3854; Sigma-Aldrich). After three washes with Tris-buffered saline containing $0.05 \%$ Tween-20 for 10 minutes each, the membranes were incubated with secondary antibodies (horseradish peroxidase-conjugated goat antirabbit IgG) for 2 hours. They were then washed three more times for 10 minutes each with Tris-buffered saline containing $0.05 \%$ Tween-20 each ( $\mathrm{pH} 7.5$ ). For visualization, horseradish peroxidase-conjugated secondary antibodies were used, and the bands were developed by Western Bright ECL substrate (Advansta, San Jose, CA). Specific bands were quantified using the Bio-Rad ChemiDoc MP Imaging system (ChemiDoc XRS; Bio-Rad Laboratories, Hercules, CA).

\section{Image Analysis}

To analyze the positive immunostaining ratio of cells, images of immunofluorescent sections (TUNEL and
Ki-67), Oil Red O staining, and immumohistochemical sections (CD4 and Ly6g) were processed by ImageJ software version 1.53a (NIH, Bethesda, MD; http:// imagej.nih.gov/ij) with Java oftware version 1.8.0_112 (64-bit; Oracle, Redwood City, CA). ImageJ software was also used to determine the fragment size of myoepithelial cells according to a previous protocol. ${ }^{19}$ Images of immunofluorescent sections (F4/80) were quantified by the microscope's analysis software (NIS6 Elements version 4.1; Nikon, Tokyo, Japan). Each sample was analyzed in at least three different areas.

\section{Statistical Analysis}

Quantitative data are presented as means \pm SD. Independent sample $t$-test and one-way analysis of variance were applied to evaluate significance between groups, and $P<0.05$ was considered statistically significant. The statistical analysis was performed with Prism software version 8.0 (GraphPad Software, La Jolla, CA).

\section{Results}

Tear Secretion Decreases in HFD Mice

HFD caused a more rapid and larger weight gain between 1 and 4 months than in their age-matched 
A

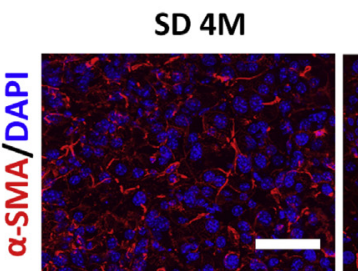

HFD 1M

HFD 2M

HFD 3M
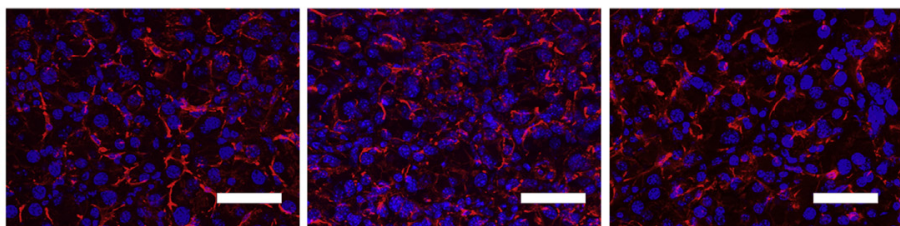

HFD 4M
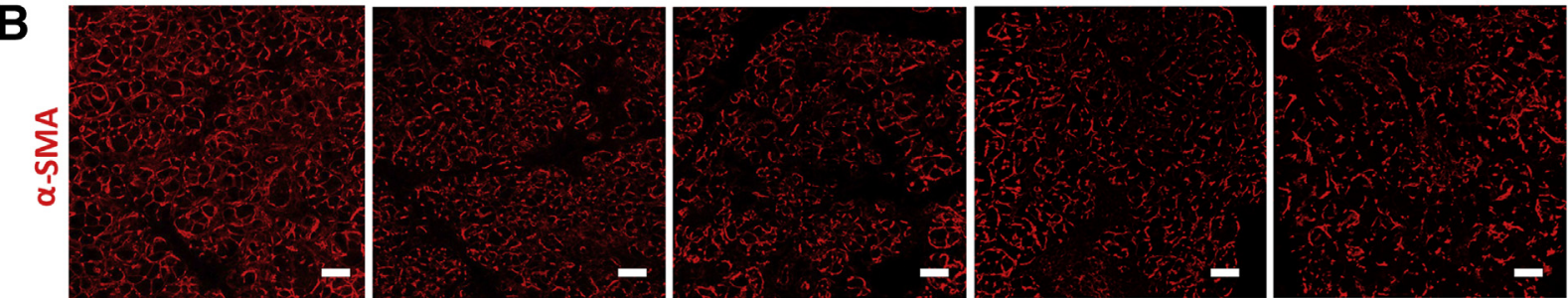

C

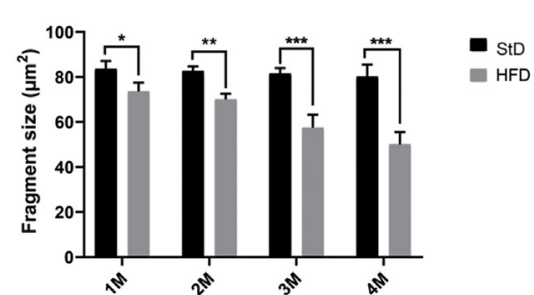

D
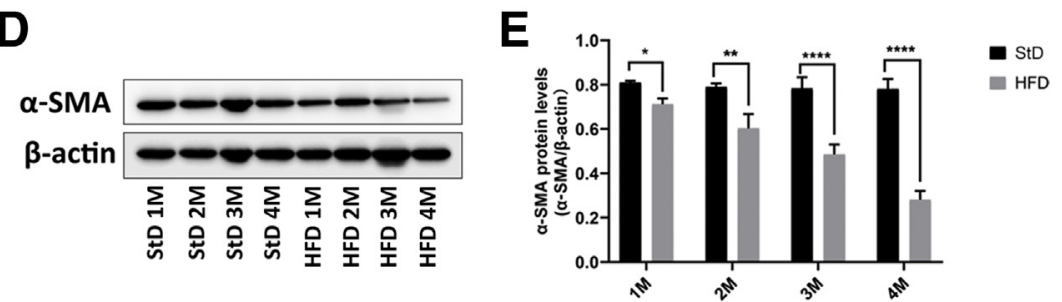

Figure 5 Myoepithelial cell structural changes of lacrimal gland in HFD mice. A: High magnification immunofluorescence staining of alpha smooth muscle actin ( $\alpha$-SMA) shows myoepithelial cell (red: $\alpha$-SMA, blue: DAPI). B: Low magnification immunofluorescence staining of alpha smooth muscle actin shows morphologic changes of the myoepithelium in the lacrimal gland (red: $\alpha$-SMA). C: ImageJ software version 1.53a was used to evaluate the average size of the stained fragments. D and E: Western blot analysis shows decreased expression of $\alpha$-SMA in the lacrimal gland of HFD mice. $n=6(\mathbf{A}-\mathbf{E})$. ${ }^{*} P<0.05$, ${ }^{*} P<0.01,{ }^{* * * P}<0.001$, and ${ }^{* * *} P<0.0001$ (one-way analysis of variance). Scale bar: $50 \mu \mathrm{m}(\mathrm{A}) ; 80 \mu \mathrm{m}$ (B). M, month; StD, standard diet.

counterparts on a StD (Figure 1A). During this interval, tear fluid secretion was consistently less in the HFD group than in the StD-fed group (Figure 1B). Furthermore, the lacrimal gland weight was higher in the HFD mice than that in the StD mice after 2 months, whereas the lacrimal gland weight fell after 3 months to a level that was lower than that in the StD mice at 4 months (Figure 1C).
A

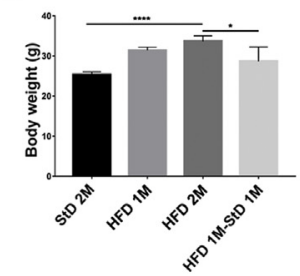

$\mathbf{E}$

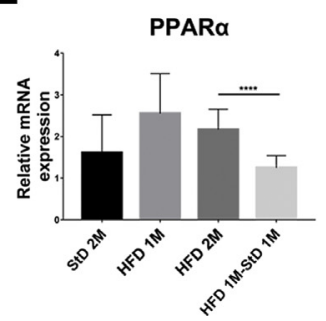

B
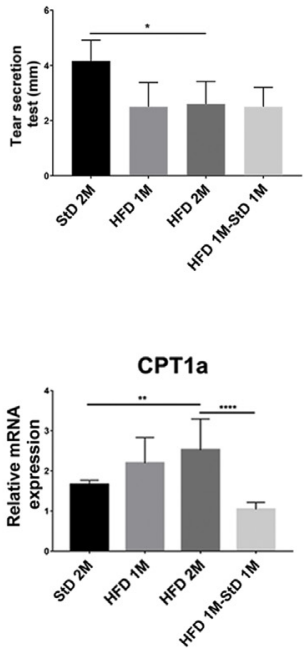

C
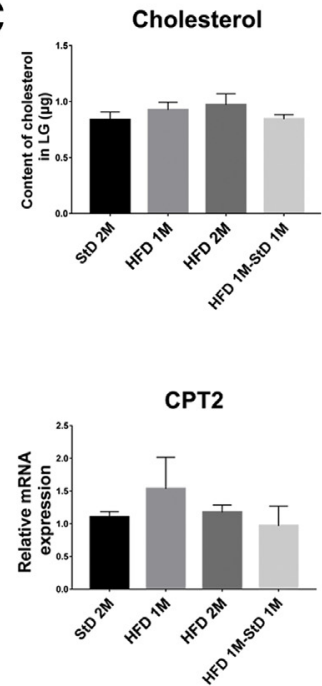

D

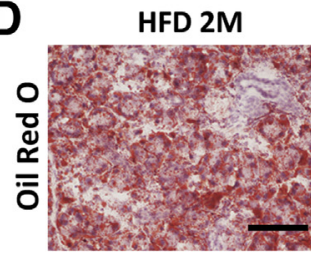

HFD 1M-StD 1M

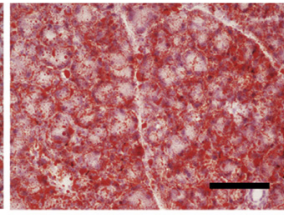

$\mathbf{F}$

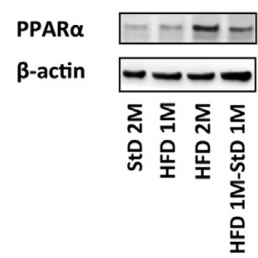

Figure 6 Standard diet (StD) shift partially reverses high-fat diet (HFD)-induced abnormal lipid metabolism in the lacrimal gland. A: Graph shows body weight. B: Tear production was evaluated by phenol red thread test. C: Content of cholesterol in the lacrimal gland was measured by cholesterol assay kit. D: 0 il Red 0 staining was used to detect lipid in the lacrimal gland. E: Real-time PCR analyzed expression of Ppar $\alpha$, Cpt1a, and Cpt2. F: Western blot results revealed the expression of Ppara in the lacrimal gland. $n=6(\mathbf{A}-\mathbf{C}, \mathbf{E}$, and $\mathbf{F}) ; n=3$ (D). ${ }^{*} P<0.05,{ }^{* *} P<0.01$, and ${ }^{* * *} P<0.0001$ (independent sample $t$-test). Scale bar $=100 \mu \mathrm{m} . \mathrm{M}$, month. 
A

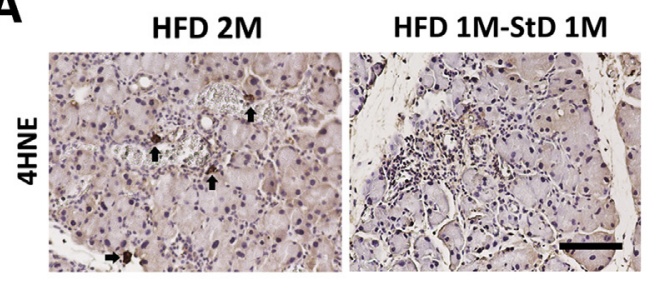

C
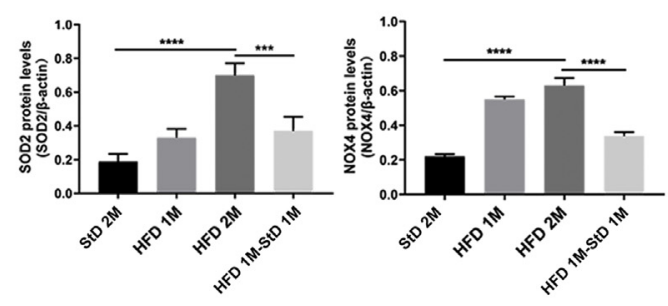

D
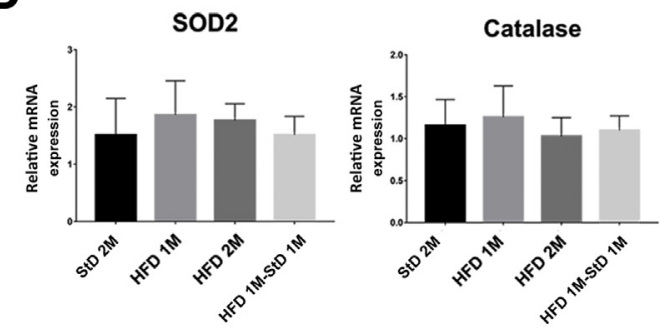

B
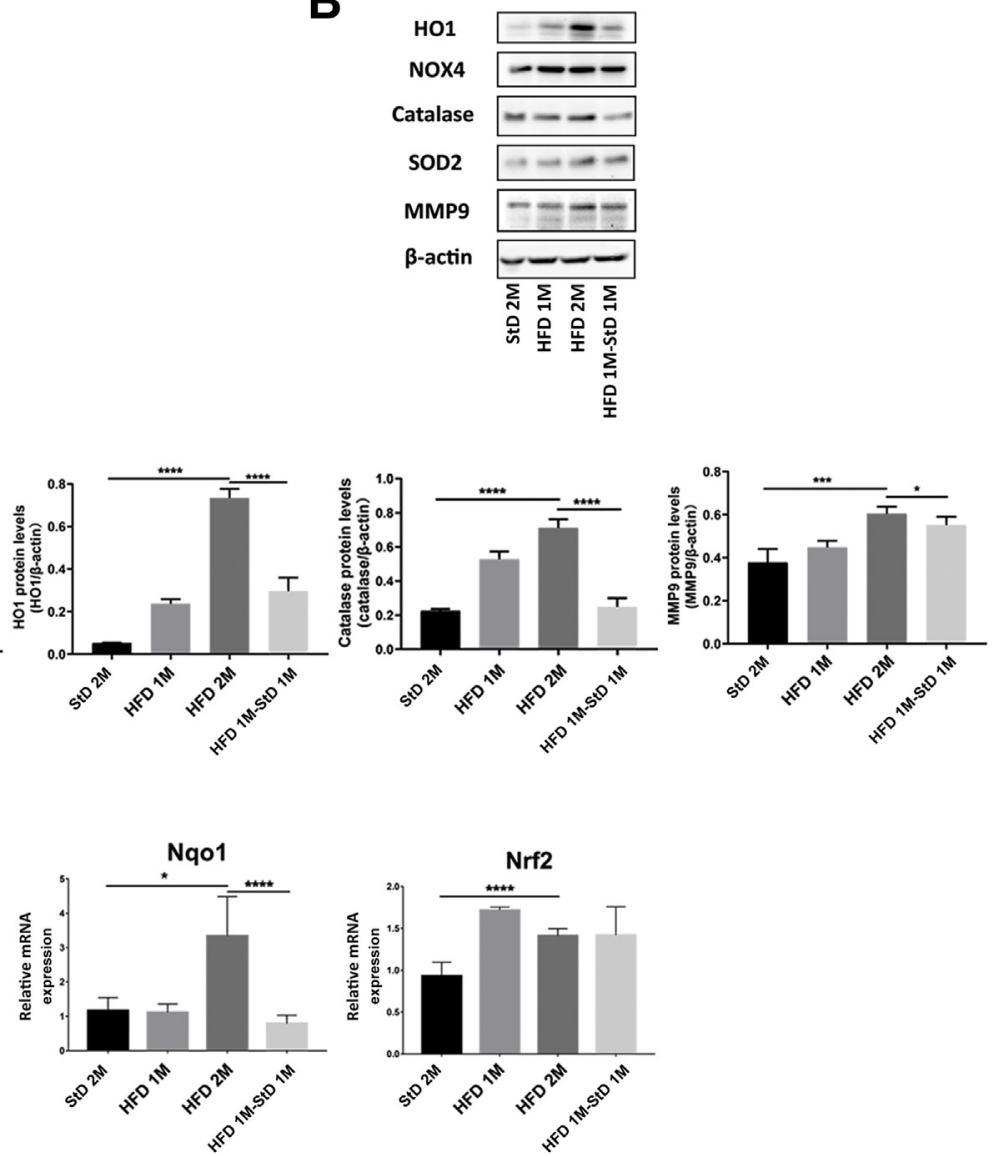

Figure 7 Standard diet (StD) shift partially reverses high-fat diet (HFD)-induced lipid peroxidation in the lacrimal gland. A: Immunohistochemical staining of $4 \mathrm{HNE}$. The arrows indicate positive staining. B and C: Western blot results revealed the expression of Ho1, Nox4, catalase, Sod2, and Mmp9. D: Real-time PCR revealed expression of Sod2, catalase, Nqo1, and Nrf2. $n=3(\mathbf{A}) ; n=6(\mathbf{B}-\mathbf{D}) .{ }^{*} P<0.05,{ }^{* *} P<0.001$, and ${ }^{* * *} P<0.0001$ (independent sample $t$-test). Scale bar $=100 \mu \mathrm{m}$. M, month.

\section{HFD Induces Abnormal Lipid Metabolism in the Lacrimal Gland}

Following 1 month on the HFD, lacrimal gland Oil Red O staining was much more pronounced in the HFD than in the StD group (Figure 1, D and G). Robust increases occurred in neutral triglyceride accumulation around the acinus. By contrast, only a few lipid droplets were evident in the basal acinar cell cytoplasm in the $\mathrm{StD}$ group (Figure 1D). This pattern of changes in the HFD mice is similar to that in the NOD mice. ${ }^{20}$ Transmission electron microscopy images of these changes confirmed that lipid droplet content dramatically increased in the cytoplasm, and as a function of time, the droplets gradually fused to cover larger areas in the cells (Figure 1E). Mitochondrial enzymatic activity plays an important role in mediating fatty acid oxidation. After the HFD for 4 months, a few mitochondrial hypermegasoma appeared (Figure 1F), which was indicative of abnormal mitochondrial metabolism often found in non-alcoholic steatohepatitis liver. ${ }^{21}$ The cholesterol content rose significantly after 2 months on the HFD and slightly less in the 4-month group than in the 3-month group (Figure 1L).
To determine whether these increases in lipid accumulation accompany altered lipid metabolism gene expression levels, real-time PCR was performed with Ppar $\alpha$, carnitine palmitoyltransferase 1a (Cpt1a) together with carnitine palmitoyltransferase 2 (Cpt2). Specifically, their expression levels rose in the early stage after initiating the HFD, whereas they remained normal or even declined after 4 months compared with the StD group (Figure 1, H, J, and $\mathrm{K})$. The decline in Ppar $\alpha$ gene expression levels was consistent with a parallel fall in its protein expression content after 4 months (Figure 1I). The increases in Cpt1a and $\mathrm{Cpt} 2$ are relevant because they play important roles in mediating translocation of long chain fatty acids across the mitochondrial membranes for beta-oxidation. ${ }^{22}$ Each of their expression levels provide readouts of Ppar $\alpha$ activation because this transcription factor induces increases in Cpt1a gene expression levels. ${ }^{23}$

\section{Lipid Peroxidation of Lacrimal Gland in HFD Mice}

Lipid peroxidation is a process in which free radical species such as peroxyl radicals, oxyl radicals, and hydroxyl radicals remove electrons from lipids and subsequently produce 


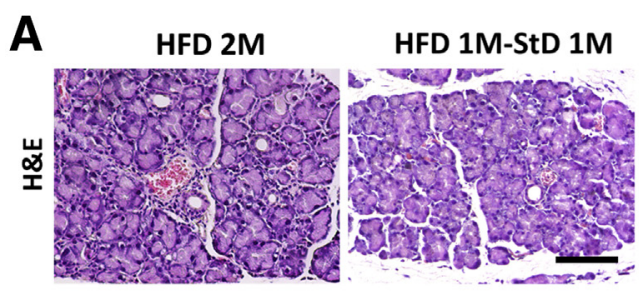

D
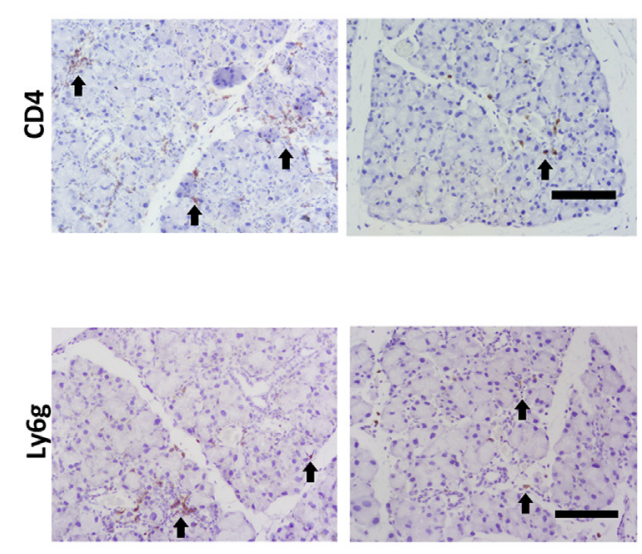

$\mathbf{F}$

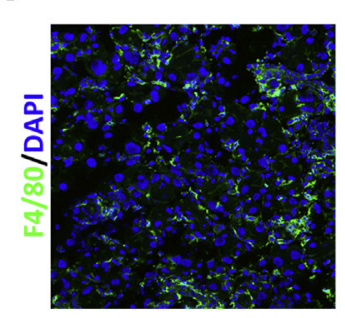

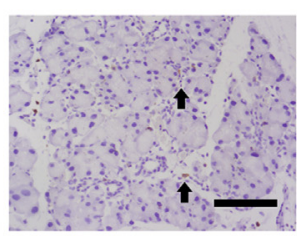

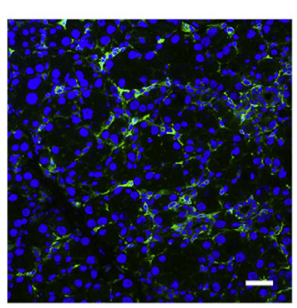

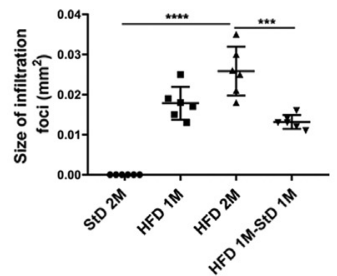
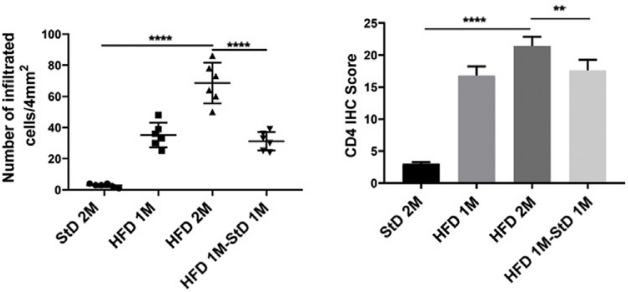

E
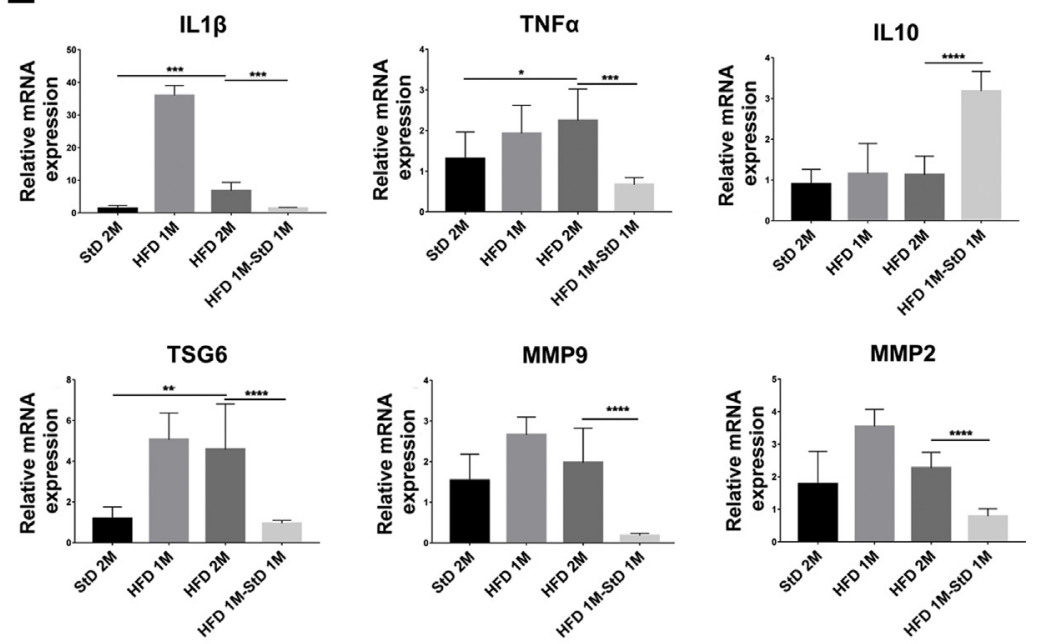

Figure 8 Standard diet (StD) shift partially reverses high-fat diet (HFD)-induced inflammation in the lacrimal gland. A: Hematoxylin and eosin (H\&E) staining reveals the morphology of the lacrimal gland. B: ImageJ software version 1.53a was used to analyze lacrimal gland infiltration size. C: ImageJ IHC Profile analysis shows a CD4-positive score. D: Immunohistochemical staining of $\mathrm{CD} 4$ displayed $\mathrm{CD}^{+} \mathrm{T}$ cells (top row) and immunohistochemical staining of Ly6g shows neutrophils in lacrimal glands (bottom row). The arrows indicate positive staining. E: Real-time PCR results show the expression of Il1 $\beta, \operatorname{Inf} \alpha, \operatorname{Il} 10$, Tsg6, Mmp9, and Mmp2. F: Immunofluorescence staining of F4/80 shows macrophages (green: F4/80, blue: DAPI). $n=6$ (A-F). ${ }^{*} P<0.05,{ }^{*} P<0.01,{ }^{* * *} P<0.001$, and ${ }_{* \star * \star} P<0.0001$ (independent sample $t$-test). Scale bar: $100 \mu \mathrm{m}$ (A and D); $40 \mu \mathrm{m}$ (F). reactive intermediates that can undergo further reactions. $^{24,25}$ Lipid peroxides can then form $4 \mathrm{HNE}$, which is a major toxic product. ${ }^{26}$ The HFD promoted $4 \mathrm{HNE}$ formation especially surrounding blood vessels (Figure 2A). MDA formation was monitored to evaluate the extent of lipid peroxidation because it is widely used as a convenient biomarker of lipid peroxidation. ${ }^{27}$ The HFD-induced MDA accumulation, which gradually rose as a function of time, was maintained at a high level after 4 months (Figure 2B). Western blot analysis showed that the HFD induced increases in oxidative stress because the Ho1, Nox4, catalase, and Sod2 oxidative stress marker expression levels dramatically increased relative to those in the StD-fed group (Figure 2, D and E). Real-time PCR results confirmed that the oxidative stress induced by the HFD was countered by rises in the gene expression levels of some anti-oxidative stress markers such as Nrf2, Nqo1, Sod2, and catalase. The expression levels of most of these markers increased before 3 months, whereas Nrf2 declined after 4 months (Figure 2C).

\section{HFD Induces Inflammatory Cell Infiltration in the Lacrimal Gland}

Following 1 month on the HFD, hematoxylin and eosin staining revealed inflammatory cell infiltration surrounding the blood vessels. This response was also evident in the intercellular space around the acini and ducts after a longer time on the HFD (Figure 3A). Immunostaining identified time-dependent increases in $\mathrm{CD}^{+} \mathrm{T}$ lymphocyte density around the acini (Figure 3, B and E). In parallel, Ly6g staining identified neutrophil infiltration around the acini after 1 month on the HFD and gradually increased as time went on (Figure 3, C and F). F4/80 staining identified gradual increases in mononuclear macrophage density after 1 month, which was sustained at a high level on the HFD to 4 months (Figure 3, D and G). The proinflammatory factors such as Il1 $\beta$ and tumor necrosis factor $\alpha(\operatorname{Tnf} \alpha)$ expression levels significantly increased in the lacrimal gland promptly after HFD feeding was initiated. The Il1 $\beta$ gene expression level was markedly up-regulated at 1 month and was higher than that in the StD group throughout 4 


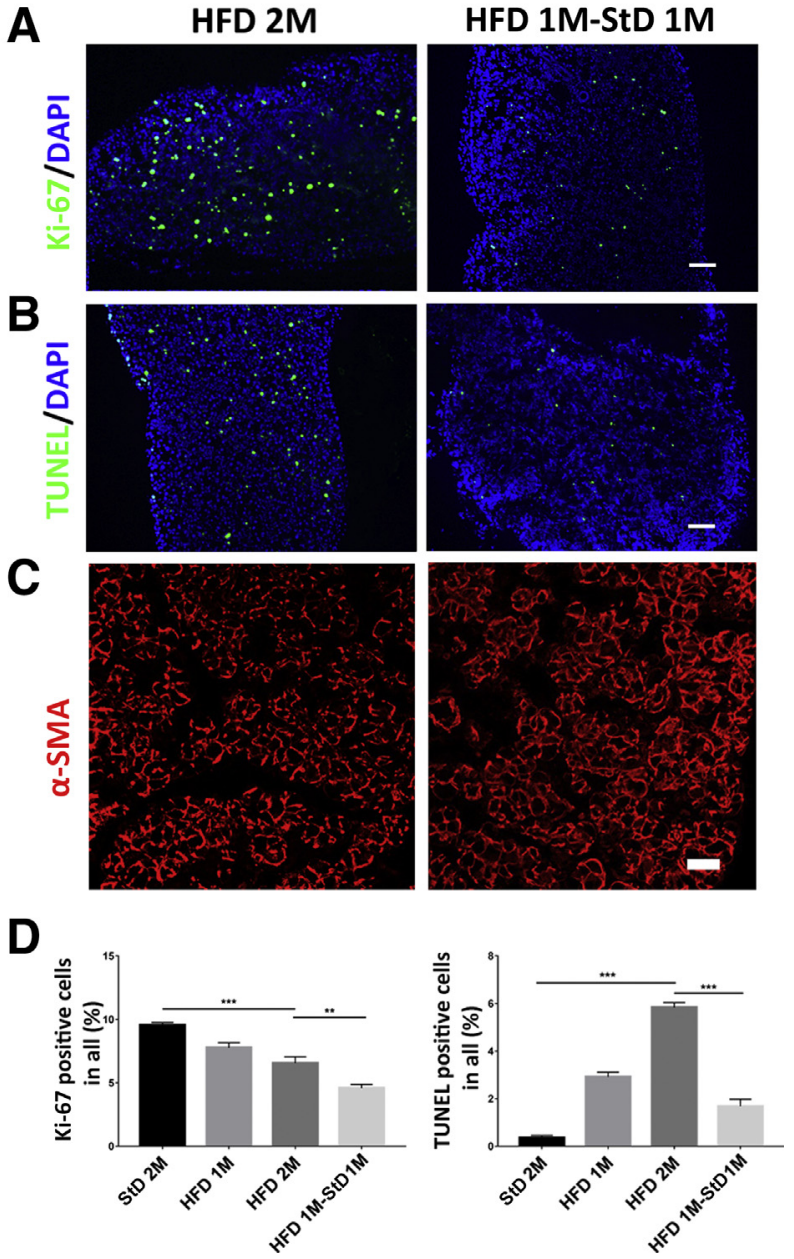

Figure 9 Standard diet (StD) shift reduces apoptosis in the lacrimal gland. A: Immunofluorescent staining of Ki-67 in the lacrimal gland (green: Ki-67, blue: DAPI). B: TUNEL staining shows apoptotic cells (green: TUNEL, blue: DAPI). C: Immunofluorescence staining of alpha smooth muscle actin shows myoepithelium morphology (red: $\alpha$-SMA). D: Graphs show quantification of Ki-67- and TUNEL-positive cells in the lacrimal gland. $n=6$ (A-D). ${ }^{* *} P<0.01,{ }^{* *} P<0.001$ (independent sample $t$-test). Scale bar: $200 \mu \mathrm{m}(\mathbf{A}$ and $\mathbf{B}) ; 80 \mu \mathrm{m}$ (C). HFD, high-fat diet; M, month; TUNEL, terminal deoxynucleotidyl transferase-mediated dUTP nick-end labeling.

months. By contrast, Tnf $\alpha$ was higher before 3 months, but after 4 months, it had fallen back to the baseline level (Figure 3H). In addition, anti-inflammatory factor Il10 expression level increased at 3 months, and the Tsg6 expression level remained high from 1 month to 4 months (Figure 3I). Increased expression of Mmp2 and -9 by lymphocytic infiltrates and epithelial cells can disturb lacrimal gland repair and secretion of aqueous tears. Gene expression of Mmp2 and Mmp9 were significantly increased at 1 month on the HFD (Figure 3J). Western blot analysis confirmed up-regulation of Mmp9 from 1 month to 3 months in the HFD mice (Figure 3K).

\section{Apoptosis and Proliferation Changes of the Lacrimal Gland in HFD Mice}

Apoptotic cells obviously increased after 1 month on the HFD and subsequently continued to rise (Figure 4A). The predominant apoptotic cells were acinar cells and myoepithelial cells instead of those in the ducts (Figure 4C). By contrast, proliferating cell abundance gradually decreased to levels that were lower than those in the StD group (Figure 4B). TUNEL-positive cell counting confirmed that HFD induced significant increases in lacrimal gland apoptosis (Figure 4D), whereas Ki-67-positive cell counting showed that the HFD inhibited cell proliferation (Figure 4D).

\section{HFD Induces Myoepithelial Cell Structural Damage in Lacrimal Gland}

$\alpha$-SMA is a contractile protein, which is a myoepithelial cell (MEC) marker. Changes in its "tentacle"-like expression modulate lacrimal gland secretory function by forming a basket-like network around the acini. ${ }^{28,29}$ After 4 months on the HFD, $\alpha$-SMA expression showed an obvious decline (Figure 5A), low magnification images demonstrated dissociation of the tentacle-like expression pattern of $\alpha$-SMA (Figure 5B), and $\alpha$-SMA-positive staining size gradually decreased from 1 month to 4 months on the HFD (Figure 5C). Western blot analysis further confirmed down-regulation of $\alpha$-SMA with HFD over time (Figure 5, D and E).

\section{StD Replacement Partially Reverses HFD-Induced Pathology in the Lacrimal Gland}

To determine whether the altered lacrimal gland structure and function were attributable to hyperlipidemia, the effects were evaluated by substituting the HFD with the StD. Accordingly, following 1 month on the HFD, mice were placed on the StD for 1 month. Their body weight decreased due to this substitution (Figure 6A). Nevertheless, this substitution was ineffective in reversing the decline of the aqueous tear secretion (Figure 6B). Lipid accumulation in the lacrimal gland only slightly decreased compared with those from mice fed the HFD for 2 months, based on cholesterol detection and Oil Red O staining (Figure 6, C and D). Furthermore, some lipid metabolism-related gene expression levels such as Ppar $\alpha$ and Cpt1a underwent down-regulation (Figure 6, E and F). Similarly, the degree of lipid peroxidation clearly decreased after the diet shift, based on declines in 4-HNE expression levels (Figure 7A). Moreover, relevant oxidative stress markers Ho1, catalase, Sod2, and Nqo1 declined (Figure 7, B-D). No increases occurred in lacrimal gland cell infiltration foci (Figure 8, A and B). Instead, inflammatory cells such as $\mathrm{CD}^{+} \mathrm{T}$ cells, neutrophils, and macrophages fell dramatically after diet replacement (Figure 8, C, D, and F, and Supplemental Figure S1). Proinflammatory factors Il1 $\beta$ and $\operatorname{Tnf} \alpha$ were down-regulated, and Il10, an antiinflammatory cytokine, was significantly up-regulated (Figure 8E). In addition, apoptotic cell abundance fell, whereas the number of proliferating cells were maintained at levels similar to those occurring in tissue isolated from mice 

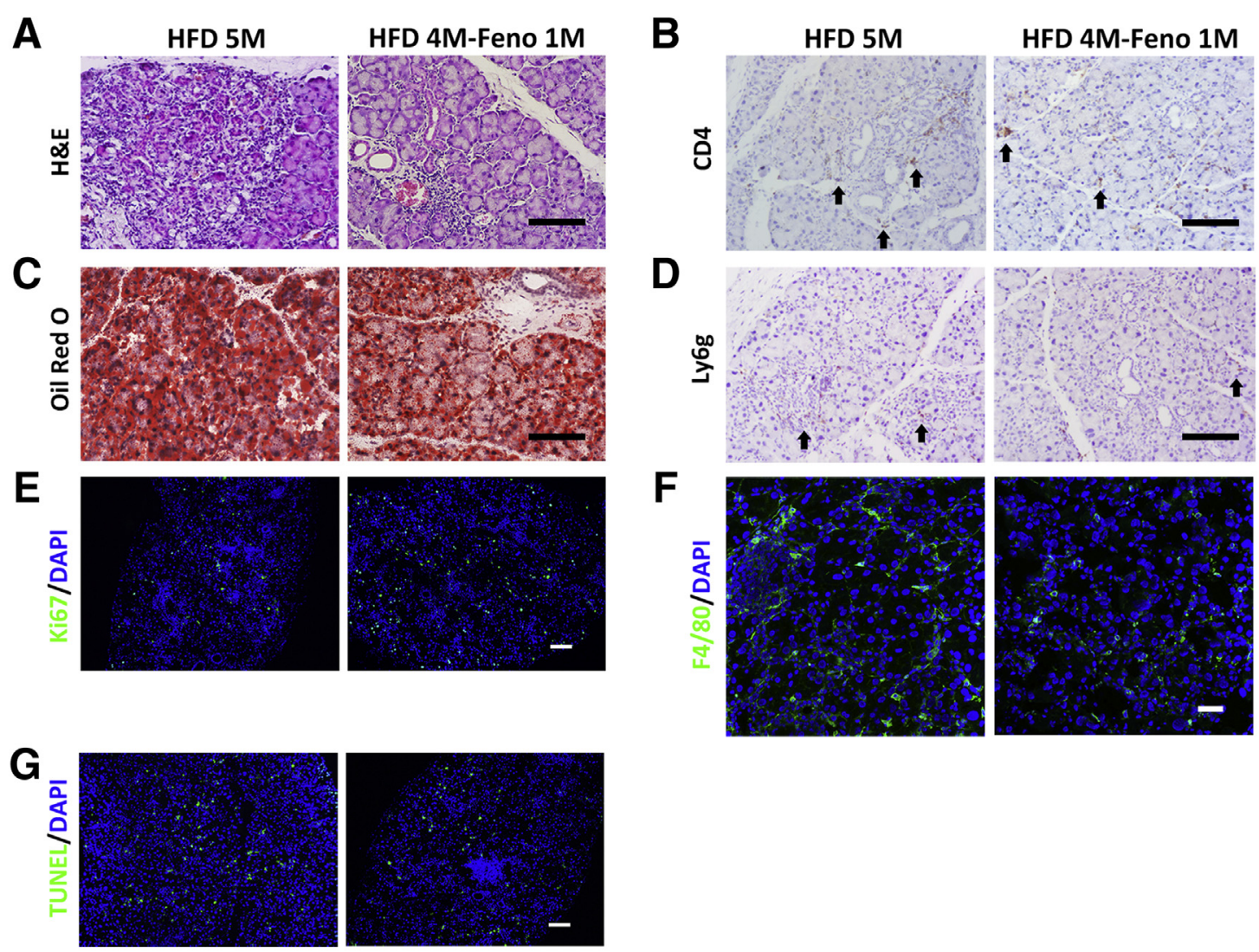

H

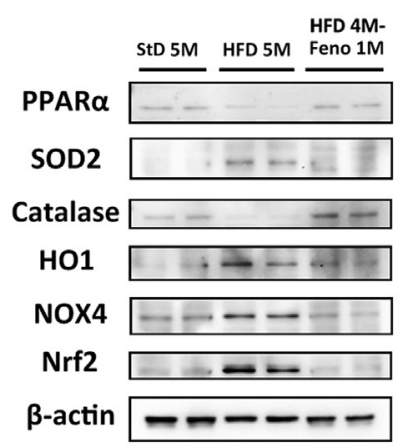

K
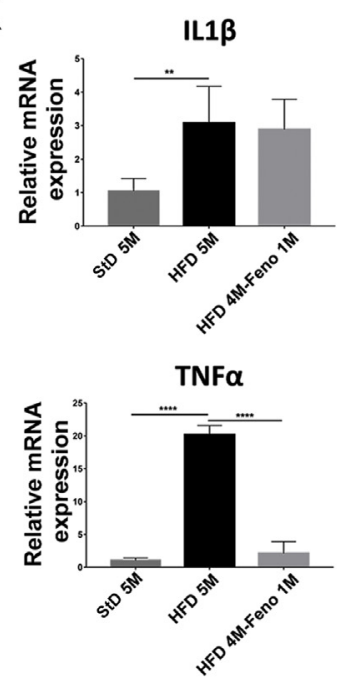
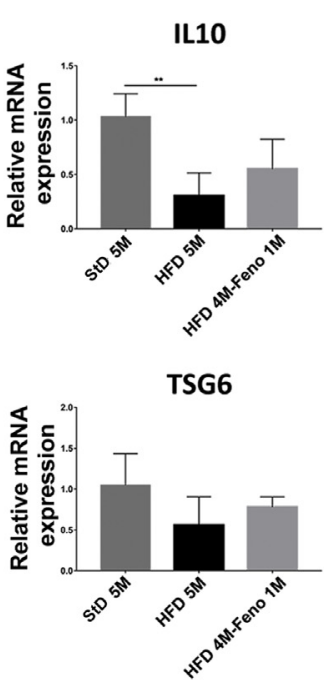

I
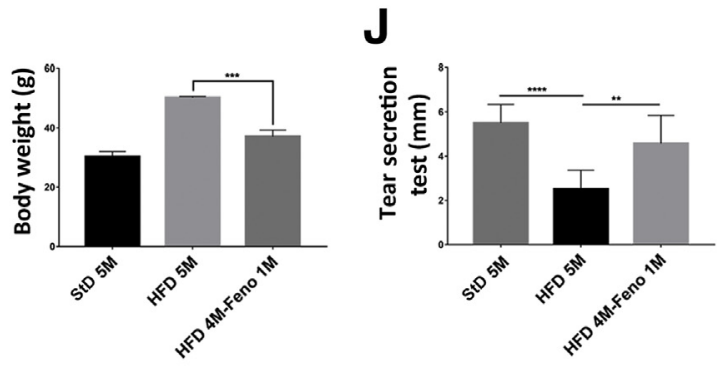
fed a StD diet (Figure 9, A, B, and D). The Mmp2 and Mmp9 expression levels declined (Figures 7, B and C, and 8E), whereas the structure of MECs showed no obvious changes based on $\alpha$-SMA staining (Figure 9C). Therefore, substituting the HFD with the StD at the very least partially reversed much of the structural and functional alterations induced by the HFD diet.

\section{Fenofibrate Reverses Lacrimal Gland Pathologic Changes Induced by Long-Term HFD}

As described above, the Ppar $\alpha$ gene expression level decreased after 4 months on the HFD. To determine whether supplementation of the HFD with a Ppar $\alpha$ agonist could offset this decline, fenofibrate was provided in the fifth month. These mice lost weight, whereas their tear secretion rose (Figure 10, I and J). After 5 months on the HFD, inflammatory cell infiltration in the lacrimal gland occurred over a large area, which was accompanied by lacrimal gland acinar atrophy. However, fenofibrate administration for 1 month after 4 months HFD significantly reduced this response and arrested further acinus atrophy (Figure 10A). Oil Red O staining declined, which was indicative of less lipid deposition (Figure 10C). Ki-67, as shown by staining, reverted back to normal levels in mice fed a $\mathrm{StD}$, and TUNEL staining showed a decrease of apoptotic cells (Figure 10, E and G, and Supplemental Figure S2D). Immunostaining showed that Ly6g-positive neutrophils, $\mathrm{CD} 4^{+}$ $\mathrm{T}$ lymphocytes, and F4/80-positive macrophages infiltrates were all significantly reduced (Figure 10, B, D, and F, and Supplemental Figure S2C). In addition, real-time PCR results showed that TNF $\alpha$ decreased significantly, whereas Il1 $\beta$ remained essentially unchanged, and Tsg6 and Il10 increased slightly. Mmp2 and Mmp9 expression levels decreased to a low level (Figure 10K). Western blot results showed that fenofibrate administration up-regulated lacrimal gland Ppar $\alpha$ expression. Antioxidative enzymatic expression levels of Ho1, Nox4, Nrf2, and Sod2 decreased significantly, whereas catalase levels rose (Figure 10H and Supplemental Figure S2, $\mathrm{A}$ and $\mathrm{B})$. Taken together, these results indicated that fenofibrate supplementation partially reversed HFD-induced declines in Ppar $\alpha$ expression levels and partially reversed lacrimal gland pathologic changes. The lacrimal gland tear secretion function was also regained after fenofibrate treatment.

\section{Discussion}

Consumption of a HFD has become increasingly common in many countries around the world and accompanies an increased risk of numerous medical conditions such as cardiovascular diseases, obesity, and metabolic syndrome. ${ }^{30}$ Such a diet increased lipid deposition in both the adipose and nonadipose tissues. Excessive accumulation of lipids in nonadipose tissues such as liver, kidney, pancreas, and heart led to cell dysfunction or even death. ${ }^{31}$ This study demonstrates that a HFD induced lipid accumulation in the lacrimal gland, which led to a series of pathologic changes in the lacrimal gland acinar that reduced lacrimal gland aqueous tear secretion.

Previous studies showed that HFD-induced lipid accumulation in the kidney could have an onset as early as at 2 months, ${ }^{32}$ whereas 3 months on this diet failed to induce lipid deposition in the salivary gland. ${ }^{33}$ In this study, lipid deposition was already apparent in the lacrimal gland after 1 month on the HFD. The rapidity of this change indicates that the lacrimal gland is more sensitive to hyperlipidemia. Ppara is one of the major regulators of lipid metabolism, which was up-regulated within 1 month after being placed on the HFD, and it was maintained at a high level for 3 months and then decreased at 4 months. This biphasic response to the HFD may be due to a compensatory response to a lipid load increase at an early stage, whereas a long-term HFD for 3 months instead down-regulated Ppar $\alpha$ expression. Ppar $\alpha$ signaling and $\mathrm{Cpt} 1 \alpha$, as well as $\mathrm{Cpt} 2$, two downstream $\beta$-oxidation-related genes, both showed similar patterns. These corresponding changes strengthen the notion that Ppar $\alpha$ may play an important role in regulating lacrimal gland lipid metabolism.

Intracellular lipid accumulation can induce oxidative stress. This response occurred on a HFD based on rises in reactive oxygen species levels and lipid peroxidation. ${ }^{34,35}$ Oxidative stress is a key factor determining the progression of numerous systemic diseases. ${ }^{36-38}$ In the current study, it is evident that the HFD activated inflammatory signaling pathways based on increases in inflammatory cell infiltration, and this response is a known indicator of oxidative stress. ${ }^{39-41}$ These effects could trigger a vicious cycle perpetuating oxidative stress and disrupting homeostasis of the internal environment in the central nerve system, skin, adipose tissue, and gut. ${ }^{42-45}$ It is presumed that increases in lipid deposition induced oxidative stress in the

Figure 10 Fenofibrate (Feno) treatment reverses lacrimal gland pathologic changes induced by long-term high-fat diet (HFD). A: Hematoxylin and eosin (H\&E) staining shows morphologic changes of the lacrimal gland. B: Immunohistochemical staining of CD4 displayed CD4 ${ }^{+}$T cells. The arrows indicate positive staining. C: Oil Red 0 staining shows lipid accumulation. D: Immunohistochemical staining of Ly6g shows neutrophils in the lacrimal gland. Arrows indicate positive staining. E: Immunofluorescent staining of Ki-67 reveals proliferative cells (green: Ki-67, blue: DAPI). F: Immunofluorescence staining of F4/80 shows macrophages (green: F4/80, blue: DAPI). G: TUNEL staining verified apoptotic cells (green: TUNEL, blue: DAPI). H: Western blot results reveal the expression of Ppar $\alpha$, Sod2, catalase, Ho1, Nox4, and Nrf2 in the lacrimal gland. I: Body weight decreases after fenofibrate treatment. J: Tear secretion was evaluated after fenofibrate treatment. K: Real-time PCR results show the expression of Il1 $\beta, \mathrm{Il} 10, \operatorname{Tnf} \alpha$, Tsg6, Mmp2, and Mmp9. $n=6(\mathbf{A}-\mathbf{K})$. ${ }^{* * P}<0.01$, ${ }^{* * * P}<0.001$, and ${ }^{* * * * P}<0.0001$ (independent sample $t$-test). Scale bar: $100 \mu \mathrm{m}(\mathbf{A}-\mathbf{D}) ; 200 \mu \mathrm{m}$ (E and $\left.\mathbf{G}\right) ; 40 \mu \mathrm{m}$ (F). M, month; StD, standard diet; TUNEL, terminal deoxynucleotidyl transferase-mediated dUTP nick-end labeling. 
lacrimal gland acinar cells and the surrounding microenvironment. On the other hand, oxidative stress activated inflammation, which in turn induced acinar cell apoptosis, as reported previously. ${ }^{46-48}$

Aqueous tear secretion decreased to about $50 \%$ of the normal level after 1 month on the HFD. This functional decline could develop as a consequence of several pathologic changes in the lacrimal gland. First, intracellular acinar cell lipid deposition inducing oxidative stress could compromise cell function. ${ }^{49,50}$ Second, increased apoptosis and decreased proliferation of acinar cells could result in a decline in lacrimal gland secretory cell mass and activity driving aqueous tear secretion. Third, as previously reported, inflammatory cell infiltration into the lacrimal gland could disrupt the acinar cells and block the ducts, which in turn obstructs aqueous tear secretion. ${ }^{10,51,52}$ Fourth, the inflammatory cytokines such as $\operatorname{Il} \beta, \operatorname{Tnf} \alpha, \operatorname{Mmp} 2$, and Mmp9 secreted by infiltrated inflammatory cells could directly reduce aqueous secretion output by the acinar cells. ${ }^{10,53}$ Lastly, MECs maintain lacrimal gland structural integrity and play an important role in inducing tear secretion. Their contractile activity generates pressure on the acinus, which promotes tear secretion. ${ }^{29,54}$ These results indicate that the MECs structure gradually became disordered and disrupted on the HFD. These changes likely reduce compression of the acinus and tear flow out of the gland. All of these pathologic changes likely help account for how the HFD reduced aqueous tear secretion.

To determine whether early intervention could reverse the pathologic changes induced by the HFD, this diet was substituted after 1 month with a StD. Indeed, lipid peroxidation, inflammation, and apoptosis were down-regulated, whereas intracellular lacrimal gland lipid accumulation was reduced slightly. Nevertheless, proliferating cells declined even further, and the tear secretion failed to increase from the low level in the HFD group. These effects indicate that HFDinduced lacrimal gland pathophysiology was irreversible during the short attempt to rescue its function. Similar phenomena were reported in sperm and liver. ${ }^{55,56}$ On the other hand, it was possible to restore normal function and reverse HFD-induced neuronal modifications by providing a normal diet for a longer time. ${ }^{57}$ Additional studies with this animal model are needed to determine whether a shift to a healthy diet for a longer period after a short-term HFD could reverse lacrimal gland decompensation.

Ppar $\alpha$ is activated by fatty acids and other lipid species, as well as by a class of chemicals referred to as peroxisome proliferators. ${ }^{58}$ In previous studies, it was found that they have tissue-specific effects on Ppara. Its expression in the substantia nigra and ventral tegmental area was reduced after 5 months on the HFD in mice, ${ }^{59}$ even though Ppar $\alpha$ was activated in the intestine after 12 weeks on the HFD in a mouse model.$^{60}$ It was suggested that at different time points while on the HFD, the expression of Ppara may be variable in various tissues. The current study found that lacrimal gland Ppar $\alpha$ expression was detectable for up to 5 months on the
HFD. In an early stage of the HFD, Ppar $\alpha$ was up-regulated from 1 month to 3 months, which is considered a positive response by the lacrimal gland to lipid accumulation. However, after a longer period on the HFD, from the fourth month to the fifth month, the lacrimal gland lost its compensatory ability to this stress because the Ppar $\alpha$ expression level started to decrease. Fenofibrate was applied to activate Ppar $\alpha$ and restore regulation of lacrimal gland lipid metabolism starting in the fourth month. It was chosen for this purpose because this agonist is extensively used to treat hypertriglyceridemia and dyslipidemia in patients. ${ }^{61-63} \mathrm{We}$ recently showed in another experimental mouse dry eye model that sleep deprivation also down-regulates Ppar $\alpha$ expression. ${ }^{64}$ The importance of Ppar $\alpha$ expression in preserving ocular surface was confirmed by showing that fenofibrate reverses lipid accumulation in the cornea ${ }^{64}$ Rises in nitric oxide generated by Ill $\beta$ were suppressed by fenofibrate-induced Ppar $\alpha$ activation in cultured lacrimal gland acinar cells. ${ }^{65}$ In the current study, fenofibrate indeed reduced inflammation, lipid deposition, and partially ameliorated declines in lacrimal gland tear secretion. These results suggest that fenofibrate may provide therapeutic benefit in dry eye induced by hyperlipidemia.

In summary, the HFD inhibited lacrimal gland fatty acid clearance based on increases in intracellular lipid accumulation. Losses in structural integrity and declines in fluid secretion are consistent with reactive oxygen species and proinflammatory cytokine up-regulation. This study provides new insight into how a lipid metabolism disorder in lacrimal gland promotes the pathogenesis underlying aqueous tear deficiency dry eye.

\section{Acknowledgment}

We thank Professor Helen P. Makarenkova (Scripps Research Institute) for her suggestions on the study.

\section{Author Contributions}

Z.L. and W.L. designed the experiments; X.H., Z.Z., S.W., J.K., M.Z., J.B., X.C., Y.L., and K.L. performed experiments; X.H., Z.Z., C.J., Z.L., and W.L. analyzed the data; X.H., P.S.R., A.J.Q., and W.L. wrote the manuscript; all authors reviewed the manuscript and gave their approval of the final version.

\section{Supplemental Data}

Supplemental material for this article can be found at http://doi.org/10.1016/j.ajpath.2020.09.002.

\section{References}

1. Paulsen F, Langer G, Hoffmann W, Berry M: Human lacrimal gland mucins. Cell Tissue Res 2004, 316:167-177 
2. Walcott B: The lacrimal gland and its veil of tears. News Physiol Sci 1998, 13:97-103

3. Zhang X, Jeyalatha MV, Qu Y, He X, Ou S, Bu J, Jia C, Wang J, Wu H, Liu Z, Li W: Dry eye management: targeting the ocular surface microenvironment. Int J Mol Sci 2017, 18:1398

4. Novack GD, Asbell P, Barabino S, Bergamini MVW, Ciolino JB, Foulks GN, Goldstein M, Lemp MA, Schrader S, Woods C, Stapleton F: TFOS DEWS II clinical trial design report. Ocul Surf 2017, 15:629-649

5. Tang SX, Lim RP, Al-Dahmash S, Blaydon SM, Cho RI, Choe CH, Connor MA, Durairaj VD, Eckstein LA, Hayek B, Langer PD, Lelli GJ, Mancini R, Rabinovich A, Servat J, Shore JW, Sokol JA, Tsirbas A, Wladis EJ, Wu AY, Shields JA, Shields C, Shinder R: Bilateral lacrimal gland disease: clinical features of 97 cases. Ophthalmology 2014, 121:2040-2046

6. Brito-Zerón P, Baldini C, Bootsma H, Bowman SJ, Jonsson R, Mariette X, Sivils K, Theander E, Tzioufas A, Ramos-Casals M: Sjögren syndrome. Nat Rev Dis Primers 2016, 2:16047

7. Yaguchi S, Ogawa Y, Kawakita T, Shimmura S, Tsubota K: Tissue reninangiotensin system in lacrimal gland fibrosis in a murine model of chronic graft-versus-host disease. Cornea 2015, 34 Suppl 11:S142-S152

8. Liu Y, Hirayama M, Kawakita T, Tsubota K: A ligation of the lacrimal excretory duct in mouse induces lacrimal gland inflammation with proliferative cells. Stem Cells Int 2017, 2017:4923426

9. Rocha EM, Alves M, Rios JD, Dartt DA: The aging lacrimal gland: changes in structure and function. Ocul Surf 2008, 6:162-174

10. Zoukhri D, Macari E, Kublin CL: A single injection of interleukin-1 induces reversible aqueous-tear deficiency, lacrimal gland inflammation, and acinar and ductal cell proliferation. Exp Eye Res 2007, 84:894-904

11. Wu K, Joffre C, Li X, MacVeigh-Aloni M, Hom M, Hwang J, Ding C, Gregoire S, Bretillon L, Zhong JF, Hamm-Alvarez SF: Altered expression of genes functioning in lipid homeostasis is associated with lipid deposition in NOD mouse lacrimal gland. Exp Eye Res 2009, 89:319-332

12. Li S, Ning K, Zhou J, Guo Y, Zhang H, Zhu Y, Zhang L, Jia C, Chen Y, Reinach PS, Liu Z, Li W: Sleep deprivation disrupts the lacrimal system and induces dry eye disease. Exp Mol Med 2018, 50: e451

13. Crispin S: Ocular lipid deposition and hyperlipoproteinaemia. Prog Retin Eye Res 2002, 21:169-224

14. Goldman JA, Julian EH: Pseudo-Sjogren syndrome with hyperlipoproteinemia. JAMA 1977, 237:1582-1584

15. Moss SE, Klein R, Klein BE: Prevalence of and risk factors for dry eye syndrome. Arch Ophthalmol 2000, 118:1264-1268

16. Wang T-J, Wang I-J, Hu C-C, Lin H-C: Comorbidities of dry eye disease: a nationwide population-based study. Acta Ophthalmol 2012, 90:663-668

17. Bu J, Wu Y, Cai X, Jiang N, Jeyalatha MV, Yu J, He X, He H, Guo Y, Zhang M, Quantock AJ, Liu Z, Wei L: Hyperlipidemia induces meibomian gland dysfunction. Ocul Surf 2019, 17:777-786

18. Livak KJ, Schmittgen TD: Analysis of relative gene expression data using real-time quantitative PCR and the $2-\Delta \Delta \mathrm{CT}$ method. Methods 2001, 25:402-408

19. Hawley D, Tang X, Zyrianova T, Shah M, Janga S, Letourneau A, Schicht M, Paulsen F, Hamm-Alvarez S, Makarenkova HP, Zoukhri D: Myoepithelial cell-driven acini contraction in response to oxytocin receptor stimulation is impaired in lacrimal glands of Sjögren's syndrome animal models. Sci Rep 2018, 8:9919

20. Ding C, MacVeigh M, Pidgeon M, da Costa SR, Wu K, HammAlvarez SF, Schechter JE: Unique ultrastructure of exorbital lacrimal glands in male NOD and BALB/c mice. Curr Eye Res 2006, 31: $13-22$

21. Caldwell SH, Swerdlow RH, Khan EM, Iezzoni JC, Hespenheide EE, Parks JK, Parker WD Jr: Mitochondrial abnormalities in nonalcoholic steatohepatitis. J Hepatol 1999, 31:430-434
22. McGarry JD, Brown NF: The mitochondrial carnitine palmitoyltransferase system. From concept to molecular analysis. Eur J Biochem 1997, 244:1-14

23. Song S, Attia RR, Connaughton S, Niesen MI, Ness GC, Elam MB, Hori RT, Cook GA, Park EA: Peroxisome proliferator activated receptor alpha (PPARalpha) and PPAR gamma coactivator (PGC-1alpha) induce carnitine palmitoyltransferase IA (CPT-1A) via independent gene elements. Mol Cell Endocrinol 2010, 325:54-63

24. Ayala A, Munoz MF, Arguelles S: Lipid peroxidation: production, metabolism, and signaling mechanisms of malondialdehyde and 4hydroxy-2-nonenal. Oxid Med Cell Longev 2014, 2014:360438

25. Su L-J, Zhang J-H, Gomez H, Murugan R, Hong X, Xu D, Jiang F, Peng Z-Y: Reactive oxygen species-induced lipid peroxidation in apoptosis, autophagy, and ferroptosis. Oxid Med Cell Longev 2019, 2019:5080843

26. Esterbauer H, Schaur RJ, Zollner H: Chemistry and biochemistry of 4-hydroxynonenal, malonaldehyde and related aldehydes. Free Radic Biol Med 1991, 11:81-128

27. Pryor WA: On the detection of lipid hydroperoxides in biological samples. Free Radic Biol Med 1989, 7:177-178

28. Wang YL, Tan Y, Satoh Y, Ono K: Morphological changes of myoepithelial cells of mouse lacrimal glands during postnatal development. Histol Histopathol 1995, 10:821-827

29. Lemullois M, Rossignol B, Mauduit P: Immunolocalization of myoepithelial cells in isolated acini of rat exorbital lacrimal gland: cellular distribution of muscarinic receptors. Biol Cell 1996, 86: $175-181$

30. Cordain L, Eaton SB, Sebastian A, Mann N, Lindeberg S, Watkins BA, O'Keefe JH, Brand-Miller J: Origins and evolution of the Western diet: health implications for the 21 st century. Am J Clin Nutr 2005, 81:341-354

31. Schaffer JE: Lipotoxicity: when tissues overeat. Curr Opin Lipidol 2003, 14:281-287

32. Yamamoto T, Takabatake $\mathrm{Y}$, Takahashi A, Kimura T, Namba T, Matsuda J, Minami S, Kaimori J-Y, Matsui I, Matsusaka T, NIImura F, Yanagita M, Isaka Y: High-fat diet-induced lysosomal dysfunction and impaired autophagic flux contribute to lipotoxicity in the kidney. J Am Soc Nephrol 2017, 28:1534-1551

33. de Carvalho PM, Gavião MBD, Carpenter GH: Altered autophagy and sympathetic innervation in salivary glands from high-fat diet mice. Arch Oral Biol 2017, 75:107-113

34. Genestra M: Oxyl radicals, redox-sensitive signalling cascades and antioxidants. Cell Signal 2007, 19:1807-1819

35. Coia H, Ma N, Hou Y, Dyba MD, Fu Y, Cruz MI, Benitez C, Graham GT, McCutcheon JN, Zheng Y-L, Sun B, Kallakury BV, Ma J, Fang H-B, Berry DL, Muralidaran V, Chung F-L: Prevention of lipid peroxidation-derived cyclic DNA adduct and mutation in highfat diet-induced hepatocarcinogenesis by Theaphenon E. Cancer Prev Res (Phila) 2018, 11:665-676

36. Dandona P, Mohanty P, Ghanim H, Aljada A, Browne R, Hamouda W, Prabhala A, Afzal A, Garg R: The suppressive effect of dietary restriction and weight loss in the obese on the generation of reactive oxygen species by leukocytes, lipid peroxidation, and protein carbonylation. J Clin Endocrinol Metab 2001, 86:355-362

37. Erhardt JG, Lim SS, Bode JC, Bode C: A diet rich in fat and poor in dietary fiber increases the in vitro formation of reactive oxygen species in human feces. J Nutr 1997, 127:706-709

38. Kobayasi R, Akamine EH, Davel AP, Rodrigues MAM, Carvalho CRO, Rossoni LV: Oxidative stress and inflammatory mediators contribute to endothelial dysfunction in high-fat dietinduced obesity in mice. J Hypertens 2010, 28:2111-2119

39. Prabhakar O: Cerebroprotective effect of resveratrol through antioxidant and anti-inflammatory effects in diabetic rats. NaunynSchmiedeberg's Arch Pharmacol 2013, 386:705-710

40. Hussain T, Tan B, Yin Y, Blachier F, Tossou MCB, Rahu N: Oxidative stress and inflammation: what polyphenols can do for us? Oxid Med Cell Longev 2016, 2016:7432797 
41. Mishra V, Banga J, Silveyra P: Oxidative stress and cellular pathways of asthma and inflammation: therapeutic strategies and pharmacological targets. Pharmacol Ther 2018, 181:169-182

42. Guillemot-Legris O, Masquelier J, Everard A, Cani PD, Alhouayek M, Muccioli GG: High-fat diet feeding differentially affects the development of inflammation in the central nervous system. J Neuroinflamm 2016, 13:206

43. Rosa DF, Sarandy MM, Novaes RD, Freitas MB, do Carmo Gouveia Pelúzio M, Gonçalves RV: High-fat diet and alcohol intake promotes inflammation and impairs skin wound healing in Wistar rats. Mediators Inflamm 2018, 2018:4658583

44. Airaksinen K, Jokkala J, Ahonen I, Auriola S, Kolehmainen M, Hanhineva K, Tiihonen K: High-fat diet, betaine, and polydextrose induce changes in adipose tissue inflammation and metabolism in C57BL/6J mice. Mol Nutr Food Res 2018, 62:e1800455

45. Guo X, Li J, Tang R, Zhang G, Zeng H, Wood RJ, Liu Z: High fat diet alters gut microbiota and the expression of paneth cellantimicrobial peptides preceding changes of circulating inflammatory cytokines. Mediators Inflamm 2017, 2017:e1800455

46. Chang C-C, Huang T-Y, Chen H-Y, Huang T-C, Lin L-C, Chang Y-J, Hsia S-M: Protective effect of melatonin against oxidative stress-induced apoptosis and enhanced autophagy in human retinal pigment epithelium cells. Oxid Med Cell Longev 2018, 2018:9015765

47. Wang S, Irving G, Jiang L, Wang H, Li M, Wang X, Han W, Xu Y, Yang Y, Zeng T, Song F, Zhao X, Xie K: Oxidative stress mediated hippocampal neuron apoptosis participated in carbon disulfideinduced rats cognitive dysfunction. Neurochem Res 2017, 42: 583-594

48. Kojima T, Wakamatsu TH, Dogru M, Ogawa Y, Igarashi A, Ibrahim OMA, Inaba T, Shimizu T, Noda S, Obata H, Nakamura S, Wakamatsu A, Shirasawa T, Shimazaki J, Negishi K, Tsubota K: Age-related dysfunction of the lacrimal gland and oxidative stress: evidence from the $\mathrm{Cu}, \mathrm{Zn}$-superoxide dismutase-1 (Sod1) knockout mice. Am J Pathol 2012, 180:1879-1896

49. Feng X, Yu W, Li X, Zhou F, Zhang W, Shen Q, Li J, Zhang C, Shen P: Apigenin, a modulator of PPAR $\gamma$, attenuates HFDinduced NAFLD by regulating hepatocyte lipid metabolism and oxidative stress via Nrf2 activation. Biochem Pharmacol 2017, $136: 136-149$

50. Spahis S, Delvin E, Borys J-M, Levy E: Oxidative stress as a critical factor in nonalcoholic fatty liver disease pathogenesis. Antioxid Redox Signal 2017, 26:519-541

51. Bron AJ, de Paiva CS, Chauhan SK, Bonini S, Gabison EE, Jain S, Knop E, Markoulli M, Ogawa Y, Perez V, Uchino Y, Yokoi N, Zoukhri D, Sullivan DA: TFOS DEWS II pathophysiology report. Ocul Surf 2017, 15:438-510

52. Damato BE, Allan D, Murray SB, Lee WR: Senile atrophy of the human lacrimal gland: the contribution of chronic inflammatory disease. Br J Ophthalmol 1984, 68:674-680
53. Aluri HS, Kublin CL, Thotakura S, Armaos H, Samizadeh M, Hawley D, Thomas WM, Leavis P, Makarenkova HP, Zoukhri D: Role of matrix metalloproteinases 2 and 9 in lacrimal gland disease in animal models of Sjögren's syndrome. Invest Ophthalmol Vis Sci $2015,56: 5218-5228$

54. Makarenkova HP, Dartt DA: Myoepithelial cells: their origin and function in lacrimal gland morphogenesis, homeostasis, and repair. Curr Mol Biol Rep 2015, 1:115-123

55. Crisóstomo L, Rato L, Jarak I, Silva BM, Raposo JF, Batterham RL, Oliveira PF, Alves MG: A switch from high-fat to normal diet does not restore sperm quality but prevents metabolic syndrome. Reproduction 2019, 158:377-387

56. Kowalski GM, Hamley S, Selathurai A, Kloehn J, De Souza DP, O'Callaghan S, Nijagal B, Tull DL, McConville MJ, Bruce CR: Reversing diet-induced metabolic dysregulation by diet switching leads to altered hepatic de novo lipogenesis and glycerolipid synthesis. Sci Rep 2016, 6:27541

57. Boitard C, Parkes SL, Cavaroc A, Tantot F, Castanon N, Layé S, Tronel S, Pacheco-Lopez G, Coutureau E, Ferreira G: Switching adolescent high-fat diet to adult control diet restores neurocognitive alterations. Front Behav Neurosci 2016, 10:225

58. Kersten S, Stienstra R: The role and regulation of the peroxisome proliferator activated receptor alpha in human liver. Biochimie 2017, $136: 75-84$

59. Kao Y-C, Wei W-Y, Tsai K-J, Wang L-C: High fat diet suppresses peroxisome proliferator-activated receptors and reduces dopaminergic neurons in the substantia nigra. Int J Mol Sci 2019, 21:207

60. Losacco MC, de Almeida CFT, Hijo AHT, Bargi-Souza P, Gama P, Nunes MT, Goulart-Silva F: High-fat diet affects gut nutrients transporters in hypo and hyperthyroid mice by PPAR-a independent mechanism. Life Sci 2018, 202:35-43

61. Gervois P, Chopin-Delannoy S, Fadel A, Dubois G, Kosykh V, Fruchart JC, Najib J, Laudet V, Staels B: Fibrates increase human REV$\mathrm{ERB} \alpha$ expression in liver via a novel peroxisome proliferator-activated receptor response element. Mol Endocrinol 1999, 13:400-409

62. Derosa G, Sahebkar A, Maffioli P: The role of various peroxisome proliferator-activated receptors and their ligands in clinical practice. J Cell Physiol 2018, 233:153-161

63. Göttlicher M, Widmark E, Li Q, Gustafsson JA: Fatty acids activate a chimera of the clofibric acid-activated receptor and the glucocorticoid receptor. Proc Natl Acad Sci U S A 1992, 89:4653-4657

64. Tang L, Wang X, Wu J, Li SM, Zhang Z, Wu S, Su T, Lin Z, Chen X, Liao X, Bai T, Qiu Y, Reinach PS, Wei L, Chen Y, Liu Z: Sleep deprivation induces dry eye through inhibition of PPAR expression in corneal epithelium. Invest Ophthalmol Vis Sci 2018, 59: 5494-5508

65. Beauregard C, Brandt PC: Peroxisome proliferator-activated receptor agonists inhibit interleukin-1beta-mediated nitric oxide production in cultured lacrimal gland acinar cells. J Ocul Pharmacol Ther 2003, 19: 579-587 